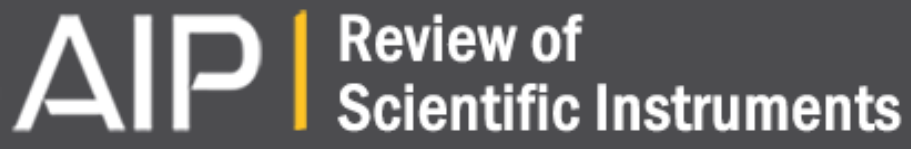

Invited Article: Dielectric material characterization techniques and designs of high-Q resonators for applications from micro to millimeter-waves frequencies applicable at room and cryogenic temperatures

Jean-Michel Le Floch, Y. Fan, Georges Humbert, Qingxiao Shan, Denis Férachou, Romain Bara-Maillet, Michel Aubourg, John G. Hartnett, Valerie Madrangeas, Dominique Cros, Jean-Marc Blondy, Jerzy Krupka, and Michael E. Tobar

Citation: Review of Scientific Instruments 85, 031301 (2014); doi: 10.1063/1.4867461

View online: http://dx.doi.org/10.1063/1.4867461

View Table of Contents: http://scitation.aip.org/content/aip/journal/rsi/85/3?ver=pdfcov

Published by the AIP Publishing

Articles you may be interested in

Hyperparametric effects in a whispering-gallery mode rutile dielectric resonator at liquid helium temperatures J. Appl. Phys. 116, 134105 (2014); 10.1063/1.4897164

A high-Q resonator using biocompatible materials at microwave frequencies

Appl. Phys. Lett. 104, 023509 (2014); 10.1063/1.4862029

Towards left-handed metamaterials using single-size dielectric resonators: The case of TiO2-disks at millimeter wavelengths

Appl. Phys. Lett. 101, 042909 (2012); 10.1063/1.4739498

Nanoliter liquid characterization by open whispering-gallery mode dielectric resonators at millimeter wave frequencies

J. Appl. Phys. 104, 074111 (2008); 10.1063/1.2991182

Dielectric property of particles at interface in random sequential adsorption and its application to whispering gallery mode resonance-shift sensors

J. Appl. Phys. 101, 023505 (2007); 10.1063/1.2422738

cerlikon

leybold vacuum

\section{online shop \\ now available \\ in 12 countries}

Vacuum Technology Made Easy

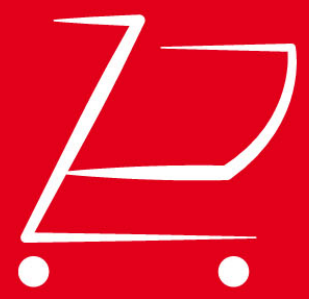

www.leyboldvacuum-shop.com 


\title{
Invited Article: Dielectric material characterization techniques and designs of high-Q resonators for applications from micro to millimeter-waves frequencies applicable at room and cryogenic temperatures
}

\author{
Jean-Michel Le Floch, ${ }^{1,2, a)}$ Y. Fan, ${ }^{1,2}$ Georges Humbert, ${ }^{3}$ Qingxiao Shan, ${ }^{1,4}$ \\ Denis Férachou, ${ }^{3,5}$ Romain Bara-Maillet, ${ }^{1,2}$ Michel Aubourg, ${ }^{3}$ John G. Hartnett, ${ }^{1,6}$ \\ Valerie Madrangeas, ${ }^{3}$ Dominique Cros, ${ }^{3}$ Jean-Marc Blondy, ${ }^{3}$ Jerzy Krupka, ${ }^{7}$ \\ and Michael E. Tobar ${ }^{1,2}$ \\ ${ }^{1}$ School of Physics, University of Western Australia, 35 Stirling Highway, Crawley, \\ Western Australia 6009, Australia \\ ${ }^{2} A R C$ Centre of Excellence, Engineered Quantum Systems (EQuS), The University of Western Australia, \\ 35 Stirling Highway, Crawley, Western Australia 6009, Australia \\ ${ }^{3}$ XLIM, UMR CNRS No 6172, 123 av. A. Thomas, 87060 Limoges Cedex, France \\ ${ }^{4}$ College of Mechatronics and Automation, National University of Defense Technology, \\ Changsha 410073, China \\ ${ }^{5}$ Department of Chemical Engineering and Biotechnology, The University of Cambridge, Cambridge CB2 3RA, \\ United Kingdom \\ ${ }^{6}$ Institute for Photonics and Advanced Sensing (IPAS) and the School of Chemistry and Physics, \\ University of Adelaide, Adelaide, S.A. 5005, Australia \\ ${ }^{7}$ Institute of Microelectronics and Optoelectronics, Warsaw University of Technology, Kosykowa 75, \\ Warsaw, Poland
}

(Received 5 March 2013; accepted 9 February 2014; published online 20 March 2014)

Dielectric resonators are key elements in many applications in micro to millimeter wave circuits, including ultra-narrow band filters and frequency-determining components for precision frequency synthesis. Distributed-layered and bulk low-loss crystalline and polycrystalline dielectric structures have become very important for building these devices. Proper design requires careful electromagnetic characterization of low-loss material properties. This includes exact simulation with precision numerical software and precise measurements of resonant modes. For example, we have developed the Whispering Gallery mode technique for microwave applications, which has now become the standard for characterizing low-loss structures. This paper will give some of the most common characterization techniques used in the micro to millimeter wave regime at room and cryogenic temperatures for designing high-Q dielectric loaded cavities. () 2014 AIP Publishing LLC. [http://dx.doi.org/10.1063/1.4867461]

\section{INTRODUCTION}

Dielectric resonators (DR) are the key element in most telecommunications such as space integrated telecommunication applications ${ }^{1-4}$ and cellular base stations, ${ }^{5-7}$ they allow a better reception and increase users for an allocated bandwidth. They are also very useful in many industries that require radar detection, proximity detection, as well as military based applications such as secure transmissions, remote guiding, navigation, and positioning systems. ${ }^{1,2}$ Also, to realize precise time and frequency references, it is necessary to design microwave sources with high spectral purity and precise frequency stability. ${ }^{8-26}$ These characteristics are directly related to the quality of the resonant element, such as cryogenic sapphire oscillators (CSOs), which are based on ultra-high-Q-factor $\left(\sim 10^{9}\right)$ sapphire DRs. These oscillators are used as a secondary frequency reference and are only oscillators of pulsing a primary standard (caesium fountain clock) at the quantum noise limit. ${ }^{10,13,27-29} \mathrm{CSOs}$ have also

\footnotetext{
a) Author to whom correspondence should be addressed. Electronic mail: jeanmichel.lefloch@uwa.edu.au
}

recently been developed to test fundamental Physics, such as a modern Michelson and Morley local Lorentz Invariance tests, using either orthogonal modes or a double dielectric sapphire resonators. ${ }^{30-32}$ The experiment searches for a difference in the speed of light in two different directions: parallel and perpendicular, the motion of the Earth around the Sun.

Depending on the application, the requirement on material properties and size of the dielectric, the make-up of the resonator can vary substantially. To make the right choice of material and dimensions, it is very important to use precise experimental and numerical techniques ${ }^{33-51}$ to properly characterize materials. In this paper, we present some important non-intrusive resonant techniques to characterize dielectric samples, which depend on the scale size of the sample. Following this, we will then describe the design of high-Q dielectric resonators using multi-layered structure such as photonic band gap and Bragg resonators at microwave and millimeter wave frequencies. These techniques of designing microwave resonators and the choice of electromagnetic mode excitations are valid and can be used from micro to millimeter wave and from millikelvin to room temperatures. 


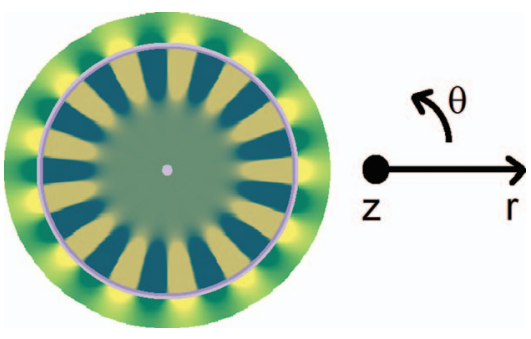

FIG. 1. Density field plot of a Whispering Gallery mode with a number of azimuthal variations $(m)$ equals to 13 . The shaded region represents the dielectric. The outer disc represents the edge of the cavity.

\section{ELECTROMAGNETIC MODES FOR MATERIAL PROPERTIES CHARACTERIZATION}

Two types of electromagnetic modes in cylindrical systems are commonly used for dielectric characterization of low-loss materials at microwave frequencies: Whispering Gallery (WG) modes and pure Transverse Electric (TE) and Transverse Magnetic (TM) modes. In spherical systems, all modes are pure TE and TM, a class of higher order modes define a greater set of modes that whisper around the surface, known as Whispering Spherical (WS) modes.

\section{A. Whispering gallery (WG) modes in cylindrical resonators}

WG modes in a cylindrical resonator may be visualized as a superposition of two rays: one moving clockwise and the other anticlockwise, propagating around the peripheral cylinder in an integer number of reflections along the $\theta$ direction (Figure 1).

In the case where the cylindrical axis ( $z$-axis) is aligned to the crystal axis, there are two different types of WG modes with electric field polarizations parallel (WGH) and perpendicular (WGE) to the axis, respectively. Each polarization has three main electromagnetic field components. WGH is defined with dominant $\mathrm{E}_{\mathrm{z}}, \mathrm{H}_{\mathrm{r}}$, and $\mathrm{H}_{\theta}$ field components and reciprocally WGE with $\mathrm{E}_{\mathrm{r}}, \mathrm{E}_{\theta}$, and $\mathrm{H}_{\mathrm{z}}$ field components, as shown in Figure 2.

The fields may be solved by using Maxwell's equations, assuming the separation of variables on the axial component of the electromagnetic field, we can write the following approximate expressions: ${ }^{51}$

$$
\begin{array}{cc}
\text { WGE mode, } E_{z}=0 & H_{z}=A_{n m} J_{n}\left(k_{r} r\right)_{\sin (m \theta)}^{\cos (m \theta)} \cos (\beta z), \\
\text { WGH mode, } H_{z}=0 & E_{z}=A_{n m} J_{n}\left(k_{r} r\right)_{\sin (m \theta)}^{\cos (m \theta)} \cos (\beta z) .
\end{array}
$$

Here $J_{n}$ is the Bessel function of order $n$, and $k_{r}$ and $k_{z}$ are the $r$ - and $z$-direction propagation constants or wave numbers in the dielectric, respectively. The other components of the field are directly calculable from the z-components using Maxwell's equations. In general in dielectric cavities, exact calculations are not possible (only approximate ${ }^{53}$ ). Thus for precise calculation numerical techniques must be used. The field plots in Figure 2 were calculated using Method of Lines. ${ }^{46,54,55}$

\section{B. Whispering spherical (WS) modes in spherical resonators}

Spherical systems possess similar modes to the cylindrical system that whisper around the surface of the sphere. We call these modes Whispering Spherical (WS) modes. General exact solutions for $E_{r}$ and $H_{r}$ field components are given by (3) and (4) for Transverse Magnetic (TM) and Transverse Electric (TE) modes, respectively:

$$
\begin{aligned}
& H_{r}=0, \quad E_{r}=n(n+1) \frac{\sqrt{k_{r} r}}{r^{2}} J_{n+\frac{1}{2}}\left(k_{r} r\right) P_{n}^{m}(\cos \phi)_{\sin (m \theta)}^{\cos (m \theta)}, \\
& E_{r}=0, \quad H_{r}=n(n+1) \frac{\sqrt{k_{r} r}}{r^{2}} J_{n+\frac{1}{2}}\left(k_{r} r\right) P_{n}^{m}(\cos \phi)_{\sin (m \theta)}^{\cos (m \theta)},
\end{aligned}
$$

where $n$ is the mode number, $k_{r}$ is the wave number and $m$ is the azimuthal mode number in the $\theta$ direction, the number of $2 \pi$ variations in the $\phi$ direction, which varies from 0 to $n$. The wave number takes discrete values corresponding to an integer number of variations in the radial direction, denoted by $p$. Modes that correspond to $p=1$ are the fundamental WS mode families. In order to find a specific solution, the composition of the system must be taken into account by solving the radial boundary value problem. In the case of spherically symmetric dielectrically loaded cavities, the dimensions of the system, permittivity of the dielectric and the shielding effect of the cavity walls set by the boundary conditions in the radial direction, leads to a complete solution.

A density plot for $n=4$ fundamental WS mode family is shown in Figure 3. We distinguish the WG mode when $m=n$ (propagation is mainly along $\theta$ direction) and Whispering Longitude (WL) mode when $\mathrm{m}=0$ (propagation is mainly along the $\phi$ direction). The rest of the modes propagate around both dimensions.

The higher the azimuthal mode number, the more the field is concentrated into the crystal and the less the
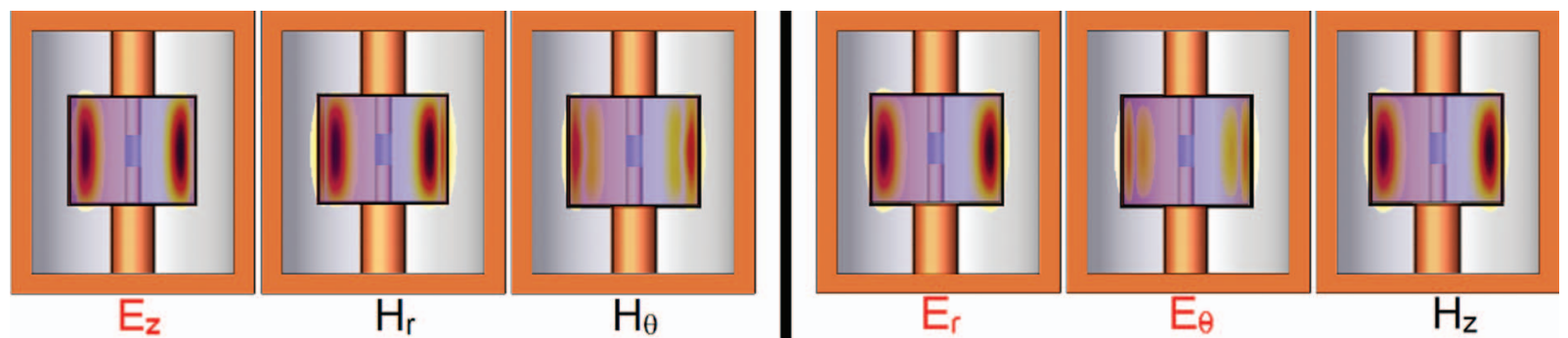

FIG. 2. (Left) Dielectric loaded cavity field density plot of a WGH or E-mode, and (right) dielectric loaded cavity density plot of a WGE or H-mode (left), as calculated using the Method of Lines. ${ }^{46,54,55}$ We chose a high azimuthal mode number to confine all the energy into the crystal illustrated with a black contour. 


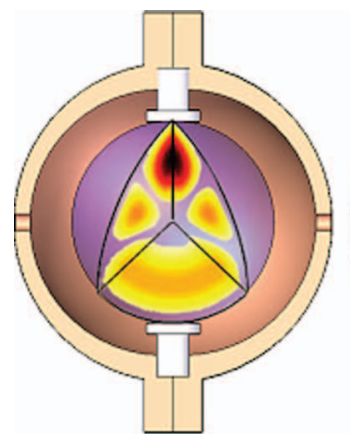

$\mathbf{m}=\mathbf{0}$

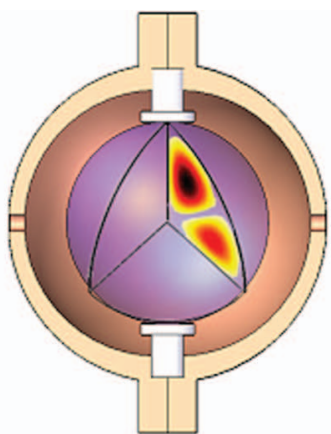

$m=1$

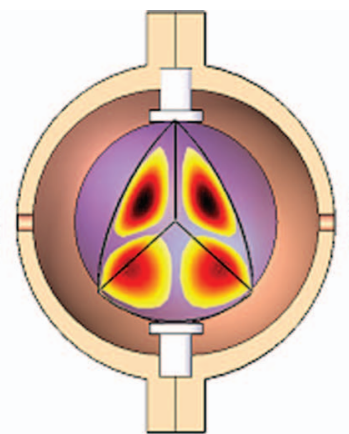

$\mathrm{m}=2$

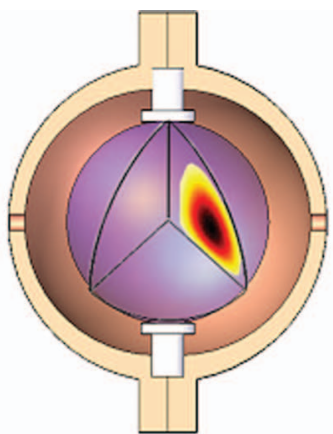

$m=3$

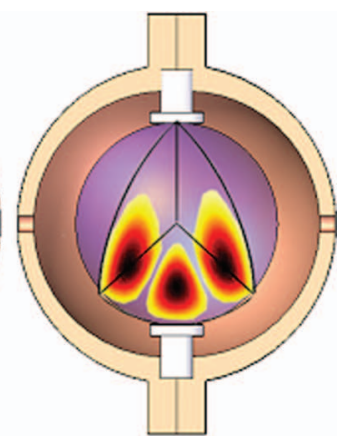

$\mathrm{m}=4$

FIG. 3. Density plot of Whispering Spherical modes for azimuthal mode numbers from $\mathrm{m}=0$ to 4 and with $n=4$.

cavity walls affect the resonator properties (except for spurious modes). The use of the whispering gallery mode permits one to make a high precision measurement of the dielectric properties using a higher order azimuthal mode numbered mode related to the high confinement of the energy in the dielectric resonator. However, there is a high density of spurious modes and for that reason we may use an open cavity. ${ }^{56-59}$ Depending on the electromagnetic mode polarization and the azimuthal mode number, caution must be taken due to spurious mode interactions which could occur between the cavity and the crystal modes. Spurious modes, mode interactions seen as a parasitic effect preventing one from obtaining the highest design performance in a resonator, or from retrieving the exact intrinsic properties of the material we would like to characterize. Therefore to overcome this problem, we may make measurements at different temperatures, where the cavity and crystal modes have different frequency dependence, or also use an open cavity structure which is done by replacing the lateral cavity wall with thin conductive posts. In the latter case, the removal of the enclosure prevents cavity modes, only Fabry-Perot type modes between the lids will remain but their presence is not so deleterious to the precise design and/or measurements sought. Another solution is to place the conducting shield as far as possible, at least a few wavelength away from the dielectric resonator and then only Whispering Gallery Modes will be excited. ${ }^{60-62}$

\section{Transverse electric and magnetic modes}

There are two types of modes shown in Figure 4, a TE and a TM. These modes have an azimuthal mode number of $m=0$. They comprise of only 3 components. Structures using these conventional modes offer better frequency isolation than the whispering gallery modes (i.e., a smaller spurious mode density). Nevertheless, their quality factor is often limited by the metallic wall losses due to lower confinement as compared to WG modes.

\section{Re-entrant cavity modes}

Microwave re-entrant cavities have been studied for over 50 years for a vast variety of applications. Development of more and more accurate structure modelling based on lumped element analysis ${ }^{63-67}$ made it very valuable for making small size high-Q resonators. These are designed for filtering applications at room and cryogenic temperatures ${ }^{68,69}$ displacement sensors for gravitational bar detectors ${ }^{70-72}$ They have also been used to develop Gunn diodes ${ }^{73}$ oscillators, electron beam tubes ${ }^{74}$ and millimetre wave resonators. ${ }^{75}$

The electromagnetic field pattern of the re-entrant cavity fundamental mode presents a very high capacitance between the internal post and the lid of the cavity, shown in Figure 5. The high electric field confinement has been useful for studying absorption process in small liquid and solid materials ${ }^{76-79}$ and also gases breakdown due to the electric E-field. ${ }^{79,80}$

\section{MATERIAL CHARACTERIZATION USING WG AND TE MODES}

We can determine the suitable electromagnetic modes for characterizing dielectric samples their thicknesses and the

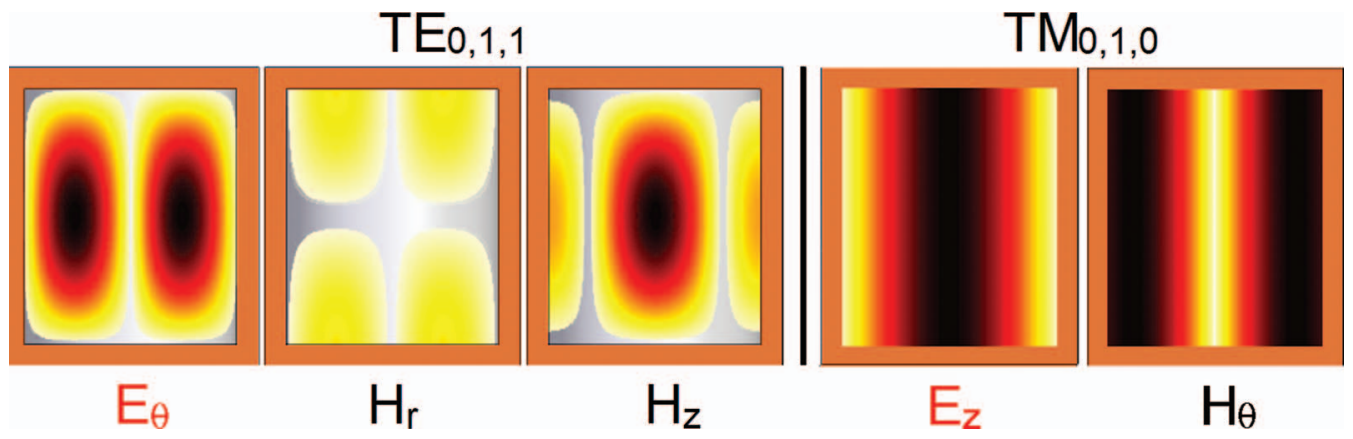

FIG. 4. Density plot of field components of a fundamental $\mathrm{TE}_{0,1,1}\left(\mathrm{E}_{\theta}, \mathrm{H}_{\mathrm{r}}, \mathrm{H}_{\mathrm{z}}\right)$ and $\mathrm{TM}_{0,1,0}\left(\mathrm{E}_{\mathrm{r}}=0, \mathrm{E}_{\mathrm{z}}, \mathrm{H}_{\theta}\right)$ mode in an empty cavity. 

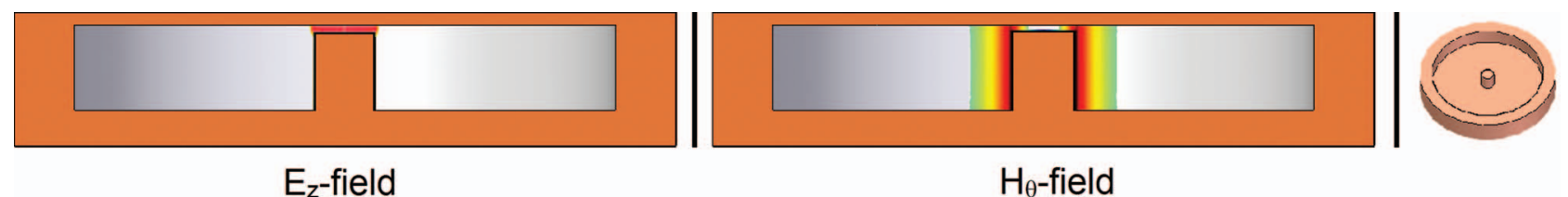

FIG. 5. Density plot of the only two field components for the fundamental re-entrant cavity mode and a 3D view of the opened cavity without lid.

resonance frequencies of interest. Depending on the sample thickness, and the resonance frequency, the Whispering Gallery mode technique may not be used if the electromagnetic modes cannot be excited into the sample. Additionally this means low loss materials are needed when using the WG measurement technique. Typically at microwave in X-band, the following techniques can be used according to the sample dimensions:

- The whispering gallery modes techniques ${ }^{15,27,47,48,55-61,81-84}$ for a thickness from $\mathrm{cm}$ to a few $\mathrm{mm}$. This is the most accurate technique to characterize low-loss material properties;

- TE modes in a metallic cavity can be used for any thickness ${ }^{35-40,44,46,49,51,53,85,86}$ and is good for measuring lossy materials or material samples below a few $\mathrm{mm}$ in thickness.

These discriminating criterions could be scaled with respect to the resonance frequencies.

Mode excitation is achieved by using probes with coaxial cables or with waveguides. The latter is more difficult to set up but this is only solution known at high millimeter-wave frequencies (Figure 6).

Commonly some field components are not used because it is harder to implement, such as $\mathrm{E}_{\theta}$ and $\mathrm{H}_{\mathrm{r}}$. A loop probe oriented either horizontally or vertically is used to excite a particular magnetic field component, $\mathrm{H}_{\mathrm{z}}$ and $\mathrm{H}_{\theta}$, respectively. A straight probe placed sideways to the cavity couples to $\mathrm{E}_{\mathrm{r}}$, from top or bottom of the cavity it couples to $E_{z}$.

\section{A. Whispering gallery mode technique}

The presented technique is valid in both topologies of Whispering Gallery modes, as shown in Figure 7.

In both cases, the sample is supported with a loaded spring system in order to hold tightly the sample and ensure a good thermal conductivity. The cavity may be closed or opened depending of the azimuthal number of the mode we excite and the losses in the dielectric sample. However, the higher the azimuthal mode number the higher the field confinement in the sample, which is quantified by the electric filling factor $\left(p_{e}\right)$. This is the fraction of electric field energy in the dielectric sample compared to the total volume of the electric energy in the cavity. The geometric factor $(G)$ is defined by the magnetic energy density applied on the metallic walls compared to the magnetic energy in the volume. Those parameters are calculated with rigorous electromagnetic simulation software, in our case it is based on the Method of Lines. ${ }^{46,54,55}$ The formulas for both $p_{e}$ and $G$ are given below in Eqs. (5) and (6) and their evolution with the azimuthal mode number in Figure 8:

$$
\begin{gathered}
G=\frac{\omega \oiiint \int_{V} \mu_{o} H \cdot H^{*} d V}{\oiint_{S} H_{t} \cdot H_{t}^{*} d S}, \\
p e=\frac{W e_{\text {material }}}{W e_{\text {Total }}}=\frac{\oiiint_{V_{\text {material }}} \varepsilon_{\text {material }} E \cdot E^{*} d V}{\oiiint_{V} \varepsilon(v) E \cdot E^{*} d V} .
\end{gathered}
$$

The general formulas to determine the intrinsic properties of the material are as following Eq. (7):

$$
\begin{aligned}
& Q_{L}^{-1}=Q_{\text {Probe1 }}^{-1}+Q_{0}^{-1}+Q_{\text {Probe2 }}^{-1} \\
& Q_{0}=Q_{L}+\left(\beta_{1}+\beta_{2}\right) Q_{L},
\end{aligned}
$$

where $\mathrm{Q}_{0}$ is the unloaded $\mathrm{Q}$-factor, $\mathrm{Q}_{\mathrm{L}}$ is the loaded $\mathrm{Q}$-factor, and $\beta_{1,2}$ the coupling factors for input and output probes, respectively.

To ensure the measurement of the intrinsic material properties, we need to be under-coupled, that means the contribution of the probes (input: (1)) and (output: (2)) to the total losses is negligible. Then the measurement given by the loaded cavity Q-factor $\left(Q_{L}\right)$ will be equal to the unloaded Qfactor $\left(Q_{o}\right)$, which is the contribution of the dielectric $(Q d)$ and the cavity wall losses $(Q m)$, Eqs. (8) and (9):

$$
\begin{gathered}
\beta \approx 0 \quad Q_{L}^{-1} \approx Q_{0}^{-1}=Q_{m}^{-1}+Q_{d}^{-1}, \\
\mathrm{Q}_{0}^{-1}=\sum_{i=1}^{N} p_{e i} \tan \delta_{i}+\frac{R_{s}}{G} .
\end{gathered}
$$

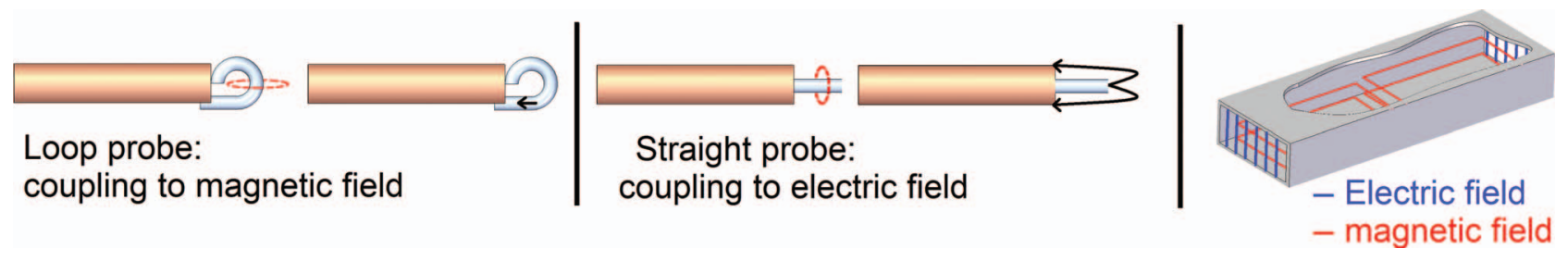

FIG. 6. Coupling methods to excite electromagnetic modes into a cavity with the associated field pattern for both coaxial cable and waveguide. 

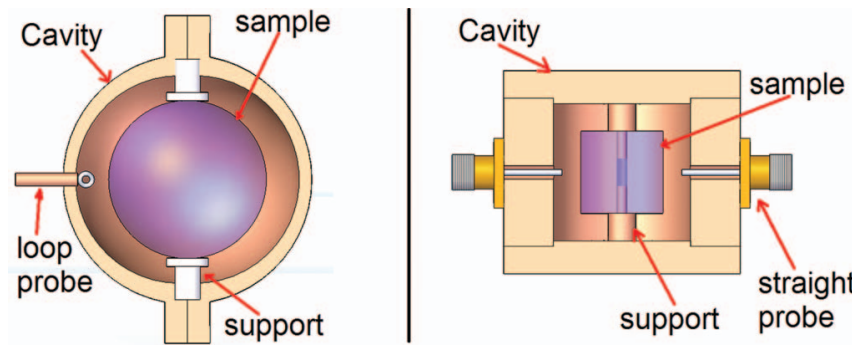

FIG. 7. (Left) Design for a spherical topology, and (right) design for a cylindrical topology to characterize the dielectric sample.

The dielectric Q-factor $Q_{d}$ is determined by the filling factor $p_{e}$, i.e., the percentage of the electric energy confined in the sample and its loss tangent. The metallic Q-factor $Q_{m}$ is given by the Geometric factor $G$ and the surface resistance $R s$ (in Ohms) of the material used to make the cavity shield, which is related to the conductivity $(\sigma)$ of the metal and the frequency $\omega / 2 \pi(\mathrm{Hz})$ of the mode we have chosen (Eq. (9)),

$$
R_{s}=\frac{1}{\sigma \delta} \quad \text { with } \quad \delta=\sqrt{\frac{2}{\omega \mu_{o} \sigma}}
$$

The determination of $R_{s}$ is obtained by measuring the empty cavity, i.e., the cavity without the sample. Then it is straight forward to deduce the loss tangent of the material. Determination of the permittivity of the sample is achieved by retrosimulation using finite element analysis, mode matching, or in our case the Method of Lines (MoL). ${ }^{46,54,55} \mathrm{MoL}$ has been used to develop rigorous electromagnetic simulation software for the purpose of material characterization; therefore, the extraction of the filling factors, geometric factor, and complex permittivity determination is obtained automatically.

The method can be used for isotropic and anisotropic material from a thickness of a few $\mathrm{cm}$ to a few $\mathrm{mm}$. This is the most accurate technique to determine the intrinsic properties of the sample by the fact that $p_{e}$ in the dielectric tends to 1 for a large azimuthal mode number. However, this method is no good for thicknesses below a few $\mathrm{mm}$, because the excitation of whispering gallery modes is not possible in samples with flat aspect ratios.

The aspect ratio of the crystal that we would like to apply the Whispering Gallery mode technique to depend on the field pattern of the electromagnetic mode and the frequency we desire. Generally, it tends to be equal close to unity (1.25). ${ }^{87}$ The larger the height, the higher is the chance of spurious modes. However, the tuning of spurious modes can be achieved by changing either the height or radius. The frequency sensitivity is greater with radial variations than that of the height. On one hand, a very small aspect ratio means a large diameter compared to height, which means the frequency, of the first fundamental mode will be lower than in the case with unity aspect ratio. On the other hand, a large aspect ratio means a small radius compared to the height, which means a higher resonance frequency for the first fundamental modes than unity aspect ratio.

Depending on the Whispering mode polarization WGH or WGE, two of the three main electromagnetic field components could be excited, i.e., $\left(\mathrm{H}_{\theta}, \mathrm{E}_{\mathrm{z}}\right)$ or $\left(\mathrm{H}_{\mathrm{z}}, \mathrm{E}_{\mathrm{r}}\right)$, respectively. The type of excitation depends on the field strength. If a field is very weak you can bring the straight probe very close to the crystal or you will need a bigger loop which could be an issue with the gap size between the cavity wall and the dielectric crystal. Reciprocally, a large field requires pulling away the straight probe or reducing the size of the loop.

\section{B. Transverse electric mode technique}

In this section, we present the technique for characterizing dielectric samples from a few $\mu \mathrm{m}$ to few $\mathrm{nm}$. The measurement is done in three steps using the fundamental transverse electric mode $\left(\mathrm{TE}_{011}\right)$. The method consists of inserting a dielectric sample through a slot into a cylindrical cavity where the electric field is maximum, and then of measuring the perturbations in frequency and Q-factor.

Step 1 consists of measuring the properties of the cavity itself. This allows us to know the conductivity of the metal and calibrate the simulation software with the measurement. We have then set the initial conditions of the measurement.

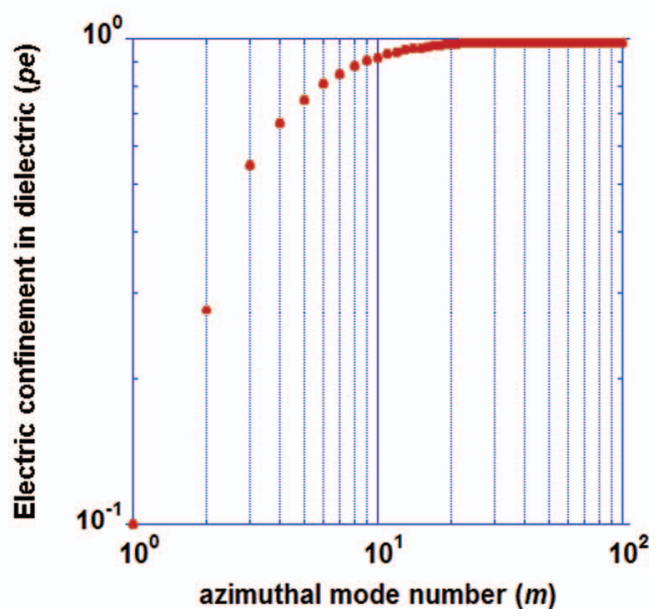

FIG. 8. (Left) Evolution of the Geometric factor $(\mathrm{G})$ vs the azimuthal mode number (m), and (right) evolution of the filling factor ( $\mathrm{p}_{\mathrm{e}}$ ) vs the azimuthal mode number $(\mathrm{m})$. 

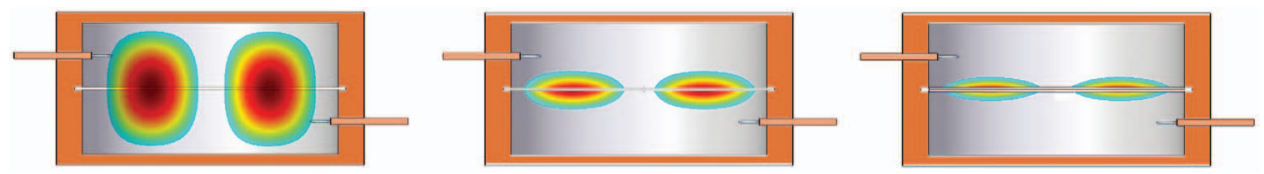

FIG. 9. Density plot of the Electric field component $\left(\mathrm{E}_{\theta}\right)$ for the three different steps of characterization superposed on the cavity design. (From left to right) Empty cavity, substrate, and substrate + deposition loaded cavities.

The initial Q-factor has to be very high in case of very low loss materials and therefore should not being limited by the cavity. This calculation is related by the equations given previously (Eqs. (6)-(9)).

For the step 2, we insert the substrate slowly to be able to track the mode frequency shift in order to deduce the right permittivity by retro-simulation. The Q-factor drop indicates the loss contribution of the dielectric, thus we can deduce the loss tangent of the substrate Eq. (11):

$$
\tan \delta=\frac{Q^{-1}-\frac{R_{S}}{G}}{p e} .
$$

Finally the step 3, we insert the same substrate sample with a deposited material of few nm. In the same way we determine the permittivity and the loss tangent of the thin film material.

This technique is not so accurate compared to Whispering Gallery mode because of a lower filling in the sample (Figure 9). However, it is the only method for thick and thin films characterization. The method is also limited to the characterization of isotropic material but is not limited by the sample thicknesses. This technique may be alternatively used for lossy material.

Also from the $60 \mathrm{~s}$, the dielectric resonator technique, for measuring permittivity and losses of low-loss dielectrics, was proposed by Hakki-Coleman. ${ }^{88}$ They used a $\mathrm{TE}_{011}$ resonant mode in a rod resonator terminated on both sides by metal conducting planes. This technique was applied by Courtney to measure more parameters, i.e., both the complex permittivity and the complex scalar permeability of microwave ferrites at frequencies greater than their ferromagnetic resonance frequency. Courtney's work benefited from a very simple measurement configuration for introducing and removing samples. The technique became known as the Courtney method ${ }^{89}$ and was proposed as one of the International Standards IEC techniques $^{90}$ for measurements of the complex permittivity of low-loss solids. For the $\mathrm{TE}_{011}$ mode, the applied electric
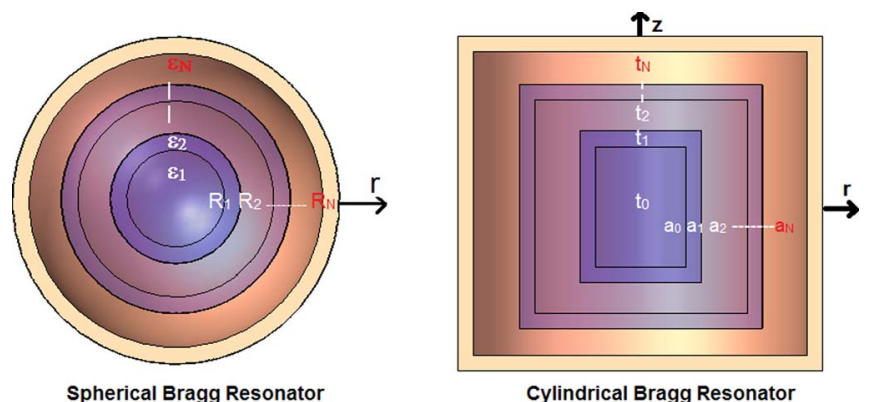

FIG. 10. Spherical and Cylindrical Bragg resonators design for $\mathrm{n}$, $\mathrm{m}$ layer structure. $\mathrm{R}_{\mathrm{i}}$ describes the radii, $\mathrm{t}_{\mathrm{i}}$ and $\mathrm{a}_{\mathrm{i}}$ the thicknesses of $i$ th layer in $\mathrm{z}$ and $\mathrm{r}$ directions. field is continuous across the sample boundary, so air gaps between the dielectric and metal planes do not play a major role. As a result, high precision measurement of the permittivity can be achieved. The Courtney resonant characterization method has its resonance frequency imposed by the dimensions and permittivity of the sample. As an example, the higher the permittivity, the lower the resonance frequency; and the thinner the sample height with small permittivity, the higher the resonance frequency becomes. The main disadvantage of the Courtney method is that the surface resistance of metal plates cannot be measured without the sample, as in cavity methods. ${ }^{91}$ This is why in our case we do not use this method. However, this difficulty has been overcome by measurements of two different modes: $\mathrm{TE}_{01 n}$ modes on the two dielectric samples made of the same material. ${ }^{92}$ Therefore, for a simple material characterization, the method becomes complex to use. In the referenced articles, they stress this is the only technique for measuring DC applied field, polarization into ferroelectrics. However, it is also possible to achieve this measurement using a resonant cavity technique. As the metal lids are separated, such resonator can be used with added DC electric field bias when the top or the bottom lid is insulated from the remaining part of resonator.

\section{DESIGN OF HIGH-Q DIELECTRIC RESONATORS USING THE BRAGG EFFECT}

The Bragg effect occurs when using one or several spherical or cylindrical reflectors. The reflector is defined by two dielectric layers of different permittivity. In our case, the reflector will be defined by a dielectric layer and a layer of freespace (Figure 10). The central region is free-space. The combination of several reflectors allows a larger concentration of field inside the low loss central region.

The Q-factor enhancement is not so significant after the addition of the first three Bragg reflectors. This is due to the dielectric loss added by the Bragg reflectors. ${ }^{12,14-18,20,93}$ The
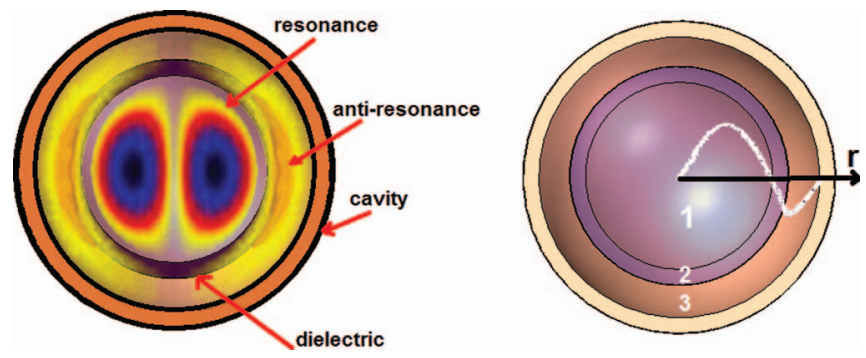

FIG. 11. (Left) Density plot of the spherical Bragg cavity where the field is highly confined in region 1 with an anti-resonance between 2 and 3, defined as a resonator. The right figure shows the simple modelling of the field pattern in $1 \mathrm{D}$. 

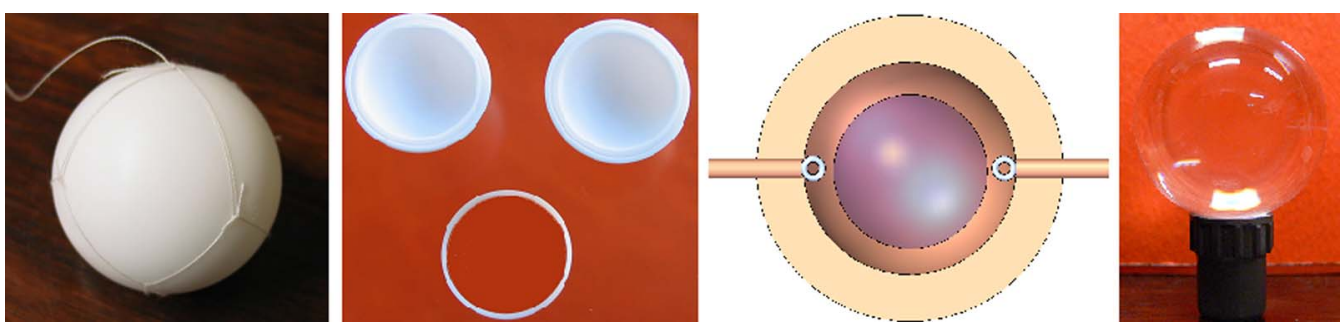

FIG. 12. Realizations of spherical Bragg resonators made with Teflon and YAG crystal (right picture).

amount of the electric field concentration achieved within the central region using this type of structure with three Bragg reflectors is about $90 \%$.

High-Q dielectric resonators using a Bragg effect have most of its importance for room temperature design compared with other structures such as Whispering Gallery mode resonators. As the field is more than $80 \%$ trapped in the free-space inner region of the Bragg structure, therefore the Q-factor does not improve consequently at cryogenic temperatures.

In the following, we summarize the work done on the design of Bragg resonators in two different topologies: spherical and cylindrical cases, respectively.

\section{A. Spherical Bragg resonator}

In order to realize a spherical Bragg resonator, we impose boundary conditions between layers. First, we study the fundamental transverse electric mode without azimuthal variation, without taking into consideration the propagating mode in the dielectric. We assume the separation of variables in spherical coordinates is valid and the field pattern can be decomposed along one propagation direction. The spherical Bessel function goes to zero at the interface of the reflector and the edge of the cavity and is maximum at the interface dielectric/free-space in the reflector, region 2 and 3 (Figures 11 and 12). The region 1 is a free space area. This is a one dimensional equation solving case. ${ }^{16,20,93}$ The left figure shows the field pattern of the fundamental Bragg mode into the spherical cavity with the anti-resonance in the reflector and resonance in the central region.
The frequency and wave number of the resonator is determined in a similar way to a mode in an empty cavity and is given by

$$
f_{o}=\frac{c k_{1}}{2 \pi} \quad \text { where } \quad k_{1}=\frac{\chi_{1}}{r_{1} \sqrt{\varepsilon_{1}}},
$$

where $\chi_{1}$ is the first root of the spherical Bessel function. The wave number also may be calculated in all the other regions subject to the "quarter wavelength" analogy. For evenly numbered regions, we calculate:

$$
k_{2 i}=\frac{\chi_{i+1}^{\prime}-\chi_{i}}{\sqrt{\varepsilon_{2 i}}\left(r_{2 i}-r_{2 i-1}\right)} \quad \text { for } \quad i=1 \text { to j. }
$$

For odd numbered regions, we calculate:

$$
k_{2 i+1}=\frac{\chi_{i+1}-\chi_{i+1}^{\prime}}{\sqrt{\varepsilon_{2 i}}\left(r_{2 i+1}-r_{2 i}\right)} \quad \text { for } \quad i=1 \text { to } \mathrm{j} .
$$

Here $j$ is the number of Bragg reflector pairs. For example, if $j$ $=1$, there is one Bragg reflector pair given by region 2 and 3 , and if $j=2$, the second pair will be given by region 4 and 5 . The reflectors must come in pairs to ensure the cancellation of the field in the reflectors. Also, $\chi_{i}^{\prime}{ }_{i}$ is the $i$ th root of the derivative of the Bessel function and $\chi_{i}$ is the $i$ th root of the Bessel function. Now, to calculate the frequency and dimensions, the wave number in all regions must be equated.

A measured Q-factor of about 22000 was achieved with a single Teflon layer Bragg resonator at $13.8 \mathrm{GHz}$ which would give us, by scaling the parameters, with a sapphire crystal, a Q-factor of about 260000 (Figure 13, Table I).

The comparison between simulation and measured results clearly shows that we have successfully demonstrated
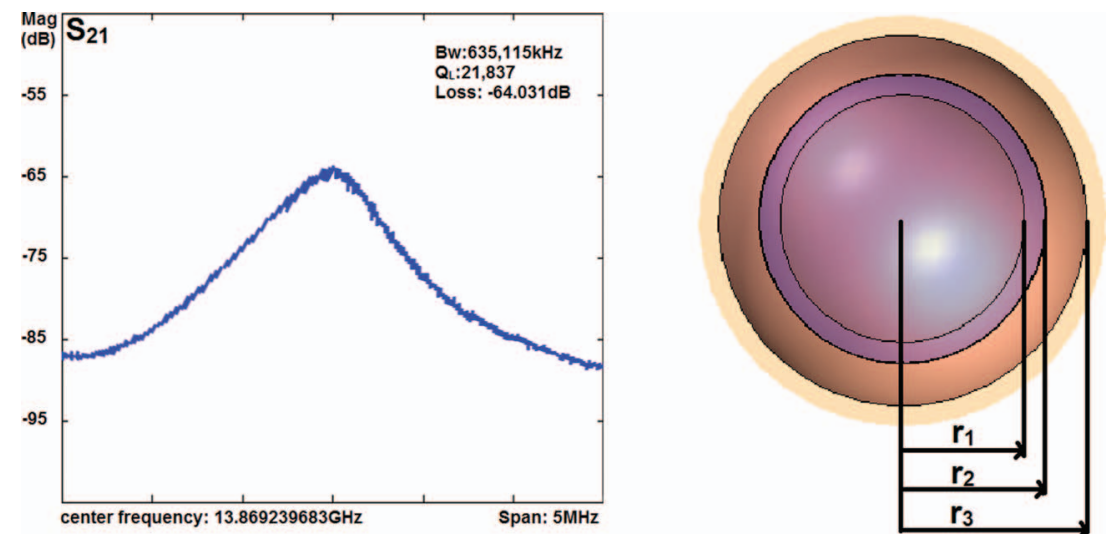

FIG. 13. (Left) Measurement in transmission at room temperature of a single Bragg spherical resonator made of Teflon, and (right) schematic for dimensions of the structure. 
TABLE I. Results from simulation and measurement of a single Bragg spherical resonator.

\begin{tabular}{lccccccccc}
\hline \hline $\mathrm{r} 1(\mathrm{~mm})$ & $\mathrm{r} 2(\mathrm{~mm})$ & $\mathrm{r} 3(\mathrm{~mm})$ & Fo (simulated) & Fo (measured) & pe1 & pe2 & pe3 & G & Qo \\
\hline 15.47 & 19.19 & 25 & $13.869 \mathrm{GHz}$ & $13.869 \mathrm{GHz}$ & 0.6942 & 0.1684 & 0.1374 & 2531.43 & 22000 \\
\hline \hline
\end{tabular}

the concept of the spherical Bragg resonator. The main limitation of the Q-factor is the dielectric loss of the Teflon. An enhancement of Q-factor was obtained with respect to a dielectric resonator limited by the loss tangent of the material. This Q-factor of 22000 is 3.5 times greater than the loss tangent limit due to the trapping of the field into the inner vacuum space.

If we replace the hollow spherical piece of Teflon by a YAG crystal, using the YAG Qf (Q-factor $x$ frequency, f) product, and the electric filling factor in the dielectric $\left(p e_{2}\right)$ we can determine the Q-factor we should obtain. The Q.f product at room temperature in the microwave regime of a YAG crystal ${ }^{47,94,95}$ is about $6 \times 10^{5}$. To evaluate the enhancement of the Q-factor, we compare the Q-factor of a bulk YAG crystal resonator (inverse of the loss tangent) with the spherical Bragg resonator in YAG. It gives an improvement of 3.9 times (Figure 14).

\section{B. Cylindrical Bragg resonator}

We have also investigated similar cylindrical Bragg resonator structures. To establish the design model, we assume the fundamental transverse electric mode. We also assume that separation of variables is valid in cylindrical coordinates so the field pattern can be decomposed in both propagation directions, $r$ and $z$, respectively. The radial direction is a Bessel function and in the axial direction; this is a sine function. Both functions go to zero at the interface of the reflector and at the edge of the cavity. They are maximum within the Bragg reflector at the interface dielectric and free-space. ${ }^{12,14,15,18}$ The density field plot shows both anti-resonance in the reflectors and the high field confinement into the central part of the resonator (Figures 15 and 16).

A linear combination of modes discovered, which links both directions with the following parameter $\gamma$

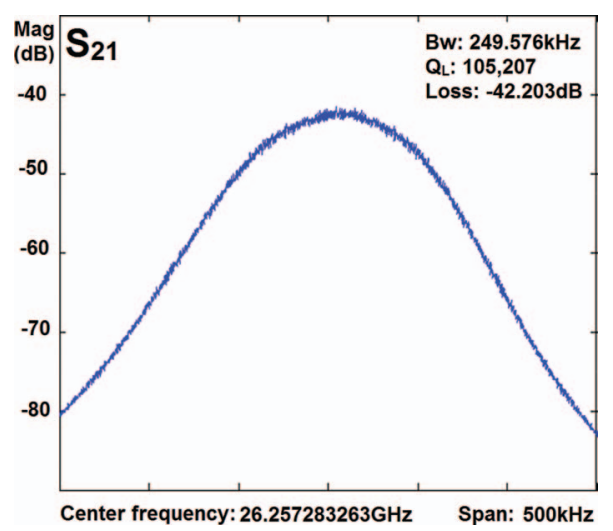

FIG. 14. Measurement in transmission at room temperature of a single Bragg spherical resonator made in crystal YAG.
(Eq. (15)):

$$
\begin{aligned}
& t_{0}=\frac{\gamma \pi}{k_{0}} \quad a_{0}=\frac{\gamma \chi_{1,1}}{k_{0} \sqrt{\gamma^{2}-1}} \\
& t_{2 i}=\frac{\pi}{2 k_{0}} \quad a_{2 j}=\frac{\gamma}{k_{0} \sqrt{\gamma^{2}-1}}\left(\chi_{1, j+1}-\chi_{1, j+1}^{\prime}\right) \\
& \mathrm{t}_{2 \mathrm{i}-1}=\frac{\pi}{2 k_{0} \sqrt{\varepsilon_{r}}} \quad a_{2 j-1}=\frac{\gamma}{k_{0} \sqrt{\varepsilon_{r} \gamma^{2}-1}}\left(\chi_{1, j+1}^{\prime}-\chi_{1, j}\right) .
\end{aligned}
$$

Equation (15) describes the thickness of each layer of the cylindrical Bragg resonator in both directions, referring to the indices $a$ and $t$ given for a multilayered distributed Bragg resonator illustrated in Figure 10. For a single Bragg resonator as shown in Figure 15, indices will become $t_{0}, t_{1}, t_{2}$ and $a_{0}$, $a_{1}, a_{2}$.

The simultaneous equations above are used to solve for $i$ axial Bragg reflectors and $j$ radial Bragg reflectors where $\mathrm{t}_{2 \mathrm{i}-1}$ and $\mathrm{a}_{2 \mathrm{j}-1}$ define the thickness of the dielectric layers of the Bragg resonator in $\mathrm{z}$ and $\mathrm{r}$ directions, respectively. A one layer cylindrical sapphire Bragg resonator with Q-factor of 230000 was achieved at $9.7 \mathrm{GHz}^{14}$ (Figures 16 and 17). This is higher than a Whispering Gallery mode resonator at room temperature at the same frequency $\left(\mathrm{Q}_{\mathrm{WGH}} \sim 200000\right.$, $\left.\mathrm{Q}_{\mathrm{WGE}} \sim 100000\right)^{83,84,96-100}$ and equivalent to a single spherical Bragg resonator. ${ }^{20}$ However, the machining and assembly are much easier in the spherical case.

During measurements, we noticed higher order confined modes existed, as shown in Figures 18 and 19.

The mode properties shown in Figure 18 has two variations in the central region and one or two in the reflectors. The Q-factor is about 94000 at $12.4 \mathrm{GHz}$ both of them, which is equivalent to a WGE mode resonator at room temperature. However, the spectrum $500 \mathrm{MHz}$ around the resonance is overmoded.

Following the modelling of simple Bragg resonator, we added a second reflector. This was made from alumina in order to prove the principle. However, the field is so confined into the centre, it has been impossible for us to couple to the high-Q mode (estimated to be 500000 ).
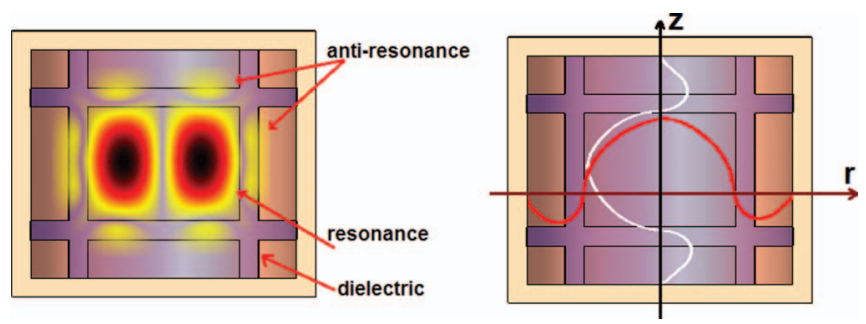

FIG. 15. (Left) Density plot of the cylindrical Bragg resonator where the field is highly confined in the centre with anti-resonance in the reflectors in both directions. (Right) It shows the simple modelling of the field pattern in $2 \mathrm{D}$ of a quarter of a structure. 

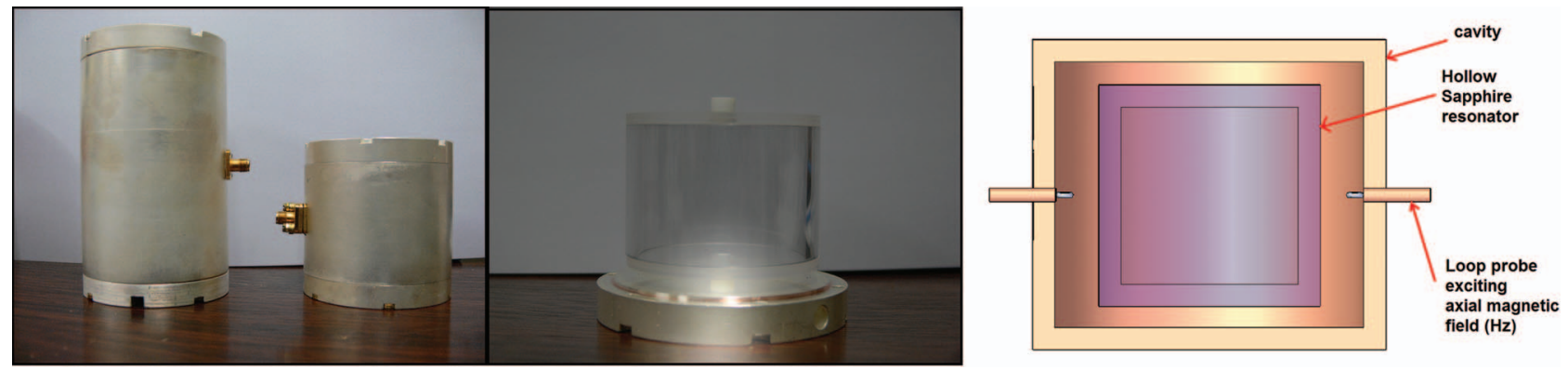

FIG. 16. Pictures of the single Bragg resonator realizations followed by a schematic of the assembly of the different pieces.
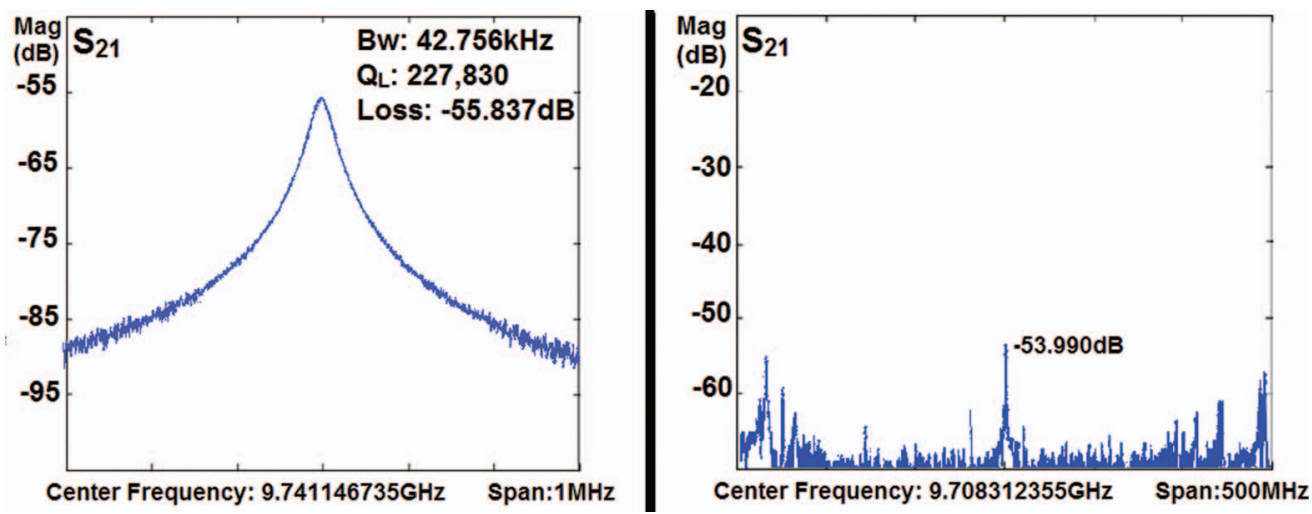

FIG. 17. (Left) Measurement with Vector Network Analyzer (VNA) in transmission $\left(\mathrm{S}_{21}\right)$ at room temperature of a single cylindrical Bragg resonator. The Q-factor measurement is given with a $10 \%$ uncertainty, value obtained directly from the VNA (right) the plot shows a clear spectrum of modes $500 \mathrm{MHz}$ around the resonance using a VNA. Data have been taken with 1601 points with a sweep time of $4 \mathrm{~s}$. We also ensured the loop excitation probes were not introducing any losses by reducing their coupling as such the loaded and unloaded Q-factors are equal.

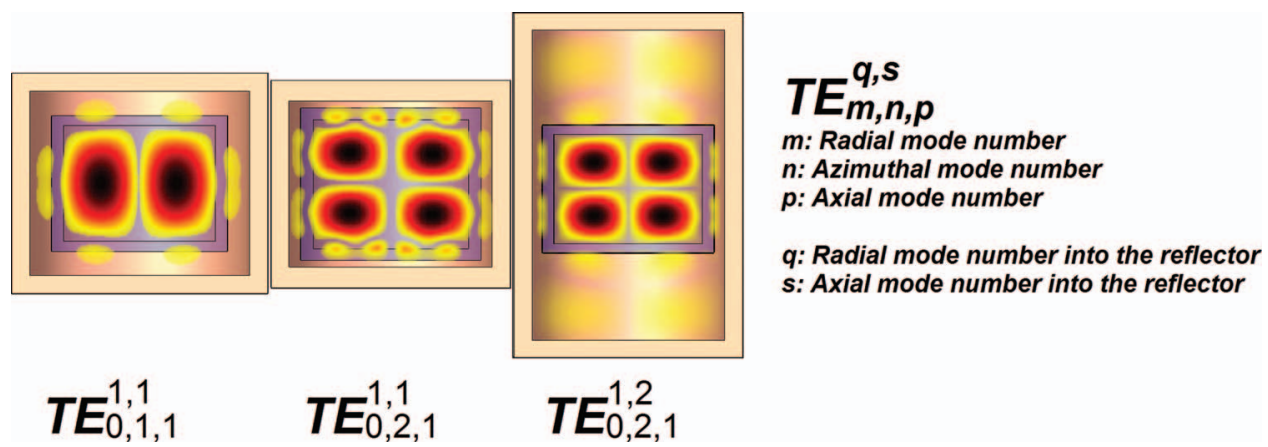

FIG. 18. Electric field density plots calculated using the method of lines software $44,51,52$ of the three different Bragg modes. The designation of each Bragg mode illustrated here is based on the electromagnetic field distribution followed by the definition of indices and exponents we use for it.
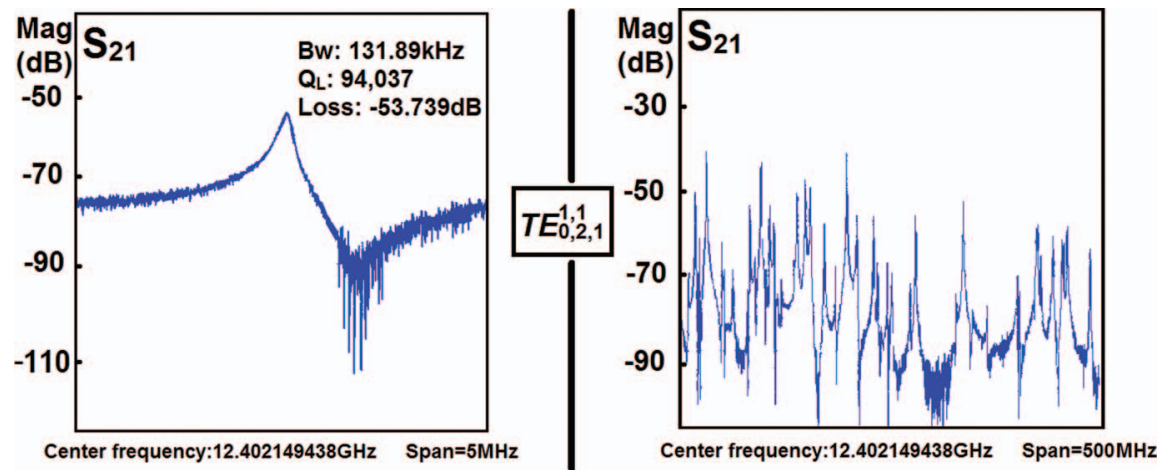

FIG. 19. (Left) Measurement in transmission at room temperature of the second Bragg mode of the Figure 18, and (right) $500 \mathrm{MHz}$ span around the resonance. 

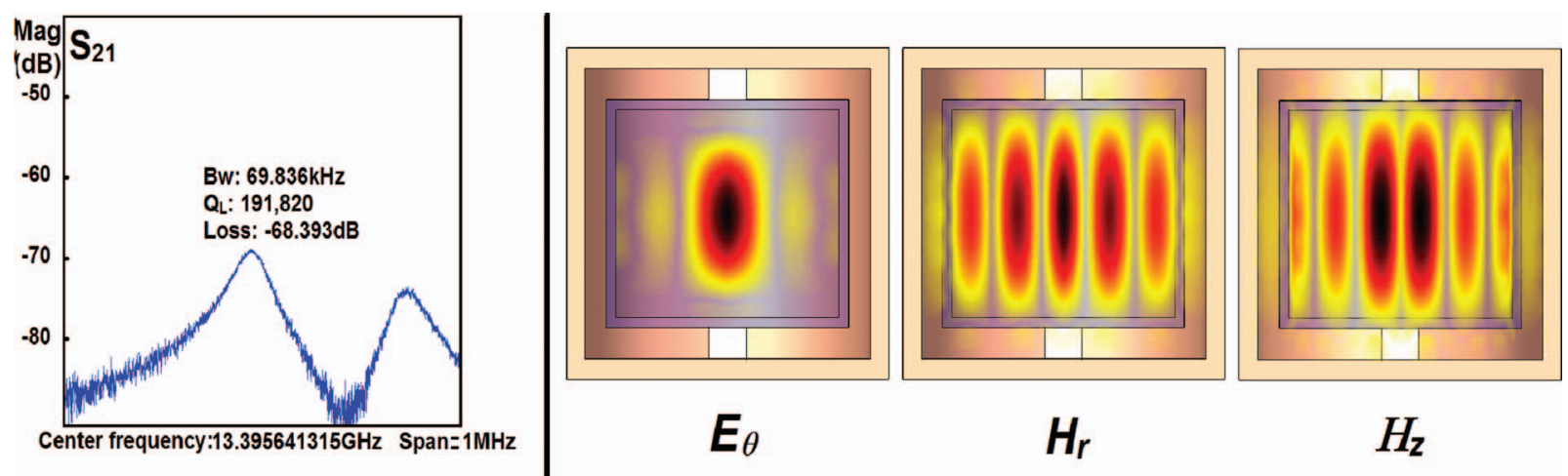

FIG. 20. (Left) Measurement in transmission at room temperature of the Hybrid Bragg mode, and (right) density plot of the 3 main electromagnetic components of this WGE and Bragg like mode.

During the characterization of the alumina, we discovered a high Q-mode at $13.4 \mathrm{GHz}$ (Figure 20) which exhibits an unloaded Q-factor of about 190000 which is six times greater than the loss tangent limit of the alumina. A retro-simulation was required to identify which type of mode it could be depending on our field excitation with the coupling probes. Then it was identified as a Hybrid yet Bragg like mode with an azimuthal number $(m)$ different to zero $(m=2)^{15}$ as illustrated in Figure 20.

\section{PHOTONIC BAND GAP RESONATOR (PBGR)- STEP TO MILLIMETRE-WAVE HIGH-Q DESIGN}

The High-Q Bragg mode design has been very successful in the microwave and lower millimetre wave frequency bands. We are now able to make Bragg resonators at different frequencies and symmetries and we also discovered an enhanced Bragg mode with azimuthal variations, which is promising at higher frequencies. However, for coverage across the entire Extremely High Frequency band, this technique is still very difficult to adapt due to the tiny dimensions as well as difficulties of coupling to the Bragg mode. Also in this frequency band, the dielectric material loss may be a problem and the choice of the right material may be limited. Diamond for example could be a good candidate ${ }^{33,101}$ for building resonators in the millimeter-wave regime. Thus, we have begun applying optical technology and concepts to the millimetre wave band. In the optical domain, hollow-core photonic crystal fibers are the most advanced manifestation of beam confinement by a photonic crystal. The beam is confined within a hollowcore by an out-of-plane 2-dimensions photonic bandgap crystal cladding that forbids field extension in the plane of the crystal. ${ }^{102}$

This principle could also work in the microwave regime if we excite an electromagnetic mode perpendicular to the crystal arrangement, the same as a Bragg mode excitation. This is called out-of-plane mode excitation. This design in microwaves will give a scaling factor to the resonator dimensions compared with standard microwave resonator structures presented in the above. We mean by a scaling factor that the size in the millimeter wave frequency band of an out-of-plane photonic bandgap resonator (PBGR) will be greater than distributed Bragg, TE-mode and WG-mode resonators. For example, we have designed an out-of-plane photonic band gap resonator (Figure 21). ${ }^{103,104}$
The simulation has been realized with the help of COMSOL software. The density plot shows the principle for 2 layers of silica rods. We have then built the PBGR resonators to prove the concept by confining the field into the centre of the structure as was done in the Bragg designs (Figure 22). The effective refractive index $\left(n_{\text {eff }}\right)$ (Eq. (16)) for an out-ofplane hollow core photonic band gap in the microwave regime is defined as

$$
n_{e f f}=\frac{\beta}{k_{\mathrm{o}}}
$$

where $\beta$ is the propagation constant (i.e., projection of the propagating vector along the propagation axis), $k_{0}$ is the wave-number in vacuum $\left(k_{0}=2 \pi \mathrm{f} / \mathrm{c}\right)$ with $\mathrm{c}$ is the speed of light in free space and $\mathrm{f}$ is the resonant frequency.

The resonator has been made with silica rods. The resonance has been found at $30 \mathrm{GHz}$, the Q-factor obtained was about 5000 with 4 layers of rods. The Q-factor for a dielectric loaded cavity depends on the electric energy filling factor, the metal surface resistance, and the geometric factor of the mode. For our case, the Q-factor is mainly limited by the metallic wall losses rather than the loss tangent.

A more compact version has been built at the same resonance frequency by optimizing the electromagnetic field confinement inside the inner region of the hexagonal metallic cavity based on out-of-plane two-dimensional photonic bandgap crystal cladding. A resonator was constructed with silica rods to prove the concept at frequencies around $30 \mathrm{GHz}$. It
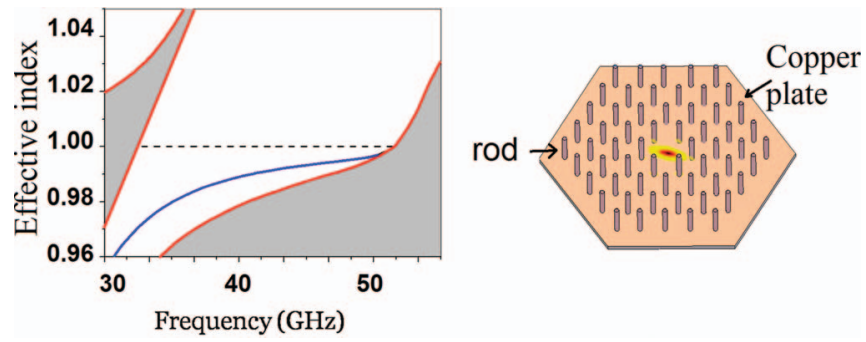

FIG. 21. Dispersion diagram of effective indices versus frequency of modes supported by the PBG crystal with one rod removed in the centre (hollowcore). ${ }^{105}$ Shaded areas delimited with plain red curves show domains where the cladding array supports modes delimiting the band gap (dash line). On the right hand side, the density plot of the fundamental mode superposed with the $3 \mathrm{D}$ view of the structure without the top lid shows where the electric field is confined in the hollow-core. 

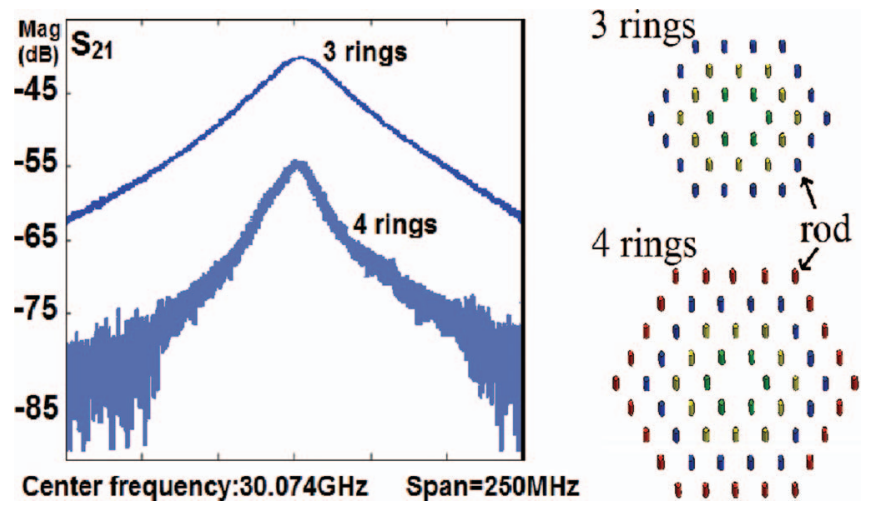

FIG. 22. Transmission measurement $\left(\mathrm{S}_{21}\right)$ at room temperature of a 3 and 4 rod-rings resonators. On the right, the assembly of 3 and 4 rings of rods distinct with a different color tone is shown.

is shown that the technique can reduce the resonator size by 8.1 times without loss in the quality factor value. ${ }^{101}$

\section{CONCLUSIONS}

This paper has reported some of the techniques achieved at microwave and millimetre wave frequencies to design high$\mathrm{Q}$ dielectric loaded resonators and to characterize material properties. We have successfully demonstrated an enhancement of Q-factor was obtained with respect to a dielectric resonator limited by the loss tangent of the material using Bragg resonator in two different topologies and could be extended to photonic band gap resonator from the optics. This was because the mode exists mainly in the inner free-space region. To improve the Q-factor of this type of resonator, a low-loss and high refractive index dielectric should be used. For improvement of the PBGR, it is necessary to limit the metal losses by confining the electromagnetic field far from the top and bottom metallic lids by placing horizontal reflectors as previously demonstrated with the Bragg resonator. High refractive index dielectric material will help enhance and optimize the Q-factor of the resonator through better field confinement in both directions.

For material characterization either we have perturbation of a resonant cavity mode with the dielectric "under test" (dUT) and we look at the frequency shift and Q-factor change (TE mode cavity for example), or we fix a resonant mode confined in the dUT under different polarizations (such as Whispering Gallery Mode), to retrieve the permittivity and loss tangent of the dUT. In the case of the proximity to spurious modes, the TE mode cavity may present a problem depending on the permittivity under investigation. In other words, if the permittivity is big enough for the resonance mode to be identifiable through the mode interaction then you will still be able to track the frequency shift. On the other hand, if the frequency shift overlaps with a nearby spurious mode, retrieving the intrinsic properties of the dUT becomes impossible with this technique. Another issue which may occur, depending on the dielectric losses affecting the mode confinement; a lossy dielectric will be better characterized with a TE mode cavity technique and an ultra-low loss dielectric with a Whispering Gallery mode cavity technique.
The characterization of materials with Bragg resonators is not very convenient for inserting bulk samples; that's the reason why no dimensions are given. However, a Bragg resonator as well as a Photonic Band Gap Resonator could be used for sensing gas. Therefore, the resonator size will be function of the gas particle resonance frequency you want to detect. Recently, the use of Bragg resonators has been investigated for detecting the spin transition in the microwave domain of an optically pumped rare earth ion.

\section{ACKNOWLEDGMENTS}

Part of this research results has been funded by the Australian Research Council, the French Research Agency (CNRS), Labex Sigma-Lim (No. ANR-10-LABX-0074-01), with the partnership between France and Australia through the Australian Academy of Science ISL Grant No. FR100027, and Le Conseil Régional du Limousin, cluster de calcul CALI. This work was also supported by the Australian Research Council Grant Nos. DP0878108, FL0992016, and CE110001013.

${ }^{1}$ PETIT: ESA project-Compact high-performance filters for space application based on dielectric resonator technology, 2013, see http://telecom.esa.int/telecom/www/object/index.cfm?fobjectid=32336.

${ }^{2} \mathrm{G}$. Wibisono and T. Firmansyah, "Design of dielectric resonators oscillator for mobile WIMAX at $2.3 \mathrm{GHz}$ with additional coupling lambda/4," in IEEE Region 10 Conference on TENCON (IEEE, 2011), pp. 489-493.

${ }^{3}$ H. Khalil, S. Bila, M. Aubourg, D. Baillargeat, S. Verdeyme, and F. Jouve, "Shape and topology optimization of microwave components," in International Conference on Advances in Computational Tools for Engineering Applications, Zouk Mosbeh, Lebanon, 15-17 July 2009, pp. 275-278.

${ }^{4}$ S. A. Vitusevich, K. Schieber, I. S. Ghosh, N. Klein, and M. Spinnler, "Design and characterization of an all-cryogenic low phase-noise sapphire K-band oscillator for satellite communication," IEEE Trans. Microwave Theory Tech. 51(1), 163-169 (2003).

${ }^{5}$ V. E. G. Walker, Ph.D. thesis, The University of Leeds, UK, 2003.

${ }^{6}$ V. Walker and I. C. Hunter, "Design of triple mode TE01 delta resonator transmission," IEEE Microwave Wireless Compon. Lett. 12(6), 215-217 (2002).

${ }^{7}$ J. F. Liang and W. D. Blair, "High Q TEO, Mode DR filters for PCS wireless base stations," IEEE Trans. Microwave Theory Tech. 46, 2493-2500 (1998).

${ }^{8}$ C. Rieck, P. Jarlemark, R. Emardson, and K. Jaldehag, "Precision of time transfer using GPS carrier phase," in Proceedings of the 22nd EFTF, Toulouse, France, 23-25 April 2008.

${ }^{9}$ F. Droz, P. Mosset, G. Barmaverain, P. Rochat, Q. Wang, M. Belloni, L. Mattioni, F. Emma, and P. Waller, "The on-board Galileo clocks: Current status and performance," in Proceedings of the 20th EFTF, Braunschweig, Germany, 27-30 March 2006.

${ }^{10}$ V. Giordano, S. Grop, B. Dubois, P-Y. Bourgeois, Y. Kersale, G. Haye, V. Dolgovskiy, N. Bulcalovic, G. Di Domenico, S. Schilt, J. Chauvin, D. Valat, and E. Rubiola, "New-generation of cryogenic sapphire microwave oscillators for space, metrology, and scientific applications," Rev. Sci. Instrum. 83, 085113 (2012).

${ }^{11}$ N. Klein, C. Zuccaro, U. Dähne, H. Schulz, N. Tellmann, R. Kutzner, A. G. Zaitsev, and R. Wördenweber, "Dielectric properties of rutile and its use in high temperature superconducting resonators," J. Appl. Phys. 78(11), 6683-6686 (1995).

${ }^{12}$ J. M. Le Floch, M. E. Tobar, D. Cros, and J. Krupka, "low-loss materials for high Q-factor microwave Bragg reflector resonators," Appl. Phys. Lett. 92, 032901 (2008)

${ }^{13}$ G. Marra, D. Henderson, and M. Oxborrow, "Frequency stability and phase noise of a pair of X-band cryogenic sapphire oscillators," Meas. Sci. Technol. 18, 1224-1228 (2007).

${ }^{14}$ J. M. Le Floch, M. E. Tobar, D. Cros, and J. Krupka, "High Q-factor distributed Bragg reflector resonators with reflectors of arbitrary thickness," IEEE Trans. Ultrason., Ferroelect., Frequency Control. 54, 2689-2695 (2007). 
${ }^{15}$ J. M. Le Floch, M. E. Tobar, and D. Mouneyrac, "Discovery of Bragg confined hybrid modes with high Q factor in a hollow dielectric resonator," Appl. Phys. Lett. 91, 142907 (2007).

${ }^{16}$ J. Krupka, M. E. Tobar, J. G. Hartnett, D. Cros, and J. M. Le Floch, "Extremely high Q-factor dielectric resonators for millimeter wave applications," IEEE Trans. Microwave Theory Tech. 53, 702-712 (2005).

${ }^{17}$ J. Krupka, A. Cwikla, R. N. Clarke, and M. E. Tobar, "High Q-factor microwave Fabry-Perot resonator with distributed Bragg reflectors," IEEE Trans. Ultrason., Ferroelect., Frequency Control. 52, 1443-1451 (2005).

${ }^{18}$ M. E. Tobar, J. M. Le Floch, D. Cros, and J. G. Hartnett, "Distributed Bragg reflector resonators with cylindrical symmetry and extremely high Q-factors," IEEE Trans. Ultrason., Ferroelect., Frequency Control. 52, 1726 (2005).

${ }^{19}$ G. Cibiel, M. Regis, O. Llopis, A. Rennane, L. Bary, R. Plana, Y. Kersale, and V. Giordano, "Optimization of an ultra low-phase noise sapphire-SiGe HBT oscillator using nonlinear CAD," IEEE Trans. Ultrason., Ferroelect., Frequency Control. 51(1), 33-41 (2004).

${ }^{20}$ M. E. Tobar, J. M. Le Floch, D. Cros, J. Krupka, J. D. Anstie, and J. G. Hartnett, "Spherical Bragg reflector resonators," IEEE Trans. Ultrason., Ferroelect., Frequency Control. 51, 1054-1059 (2004).

${ }^{21}$ A. G. Mann, C. Sheng, and A. N. Luiten, "Cryogenic sapphire oscillator with exceptionally high frequency stability," IEEE Trans. Instrum. Meas. 50(2), 519-521 (2001).

${ }^{22}$ C. A. Flory and H. L. Ko, "Microwave oscillators incorporating high performance distributed Bragg reflector resonators," IEEE Trans. Ultrason., Ferroelect., Frequency Control. 45, 824-829 (1998).

${ }^{23}$ R. Comte, S. Gendraud, and P. Guillon, "New concept for low loss microwave devices," Electron. Lett. 30, 419-420 (1994).

${ }^{24}$ D. L. Creedon, K. Benmessai, M. E. Tobar, J. G. Hartnett, P-Y. Bourgeois, Y. Kersalé, J-M. Le Floch, and V. Giordano, "High-power solid-state sapphire whispering gallery mode maser," IEEE Trans. Ultrason., Ferroelect., Frequency Control. 57(3), 641-646 (2010).

${ }^{25}$ D. G. Santiago, G. J. Dick, and A. Prata, "Mode control of cryogenic whispering-gallery mode sapphire dielectric-ring resonators," IEEE Trans. Microwave Theory Tech. 42(1), 52-55 (1994).

${ }^{26}$ M. M. Driscoll, J. T. Haynes, R. A. Jelen, R. W. Weinert, J. R. Gavaler, J. Talvacchio, G. R. Wagner, K. A. Zaki, and X.-P. Liang, "Cooled, ultrahigh Q, sapphire dielectric resonators for low-noise, microwave signal generation," IEEE Trans. Ultrason., Ferroelect., Frequency Control. 39(3), 405-411 (1992).

${ }^{27}$ J. G. Harnett, N. R. Nand, C. Wang, and J-M. Le Floch, "Cryogenic sapphire oscillator using a low-vibration design pulse-tube cryocooler: First results," IEEE Trans. Ultrason., Ferroelect., Frequency Control. 57 (5), 1034-1038 (2010).

${ }^{28}$ S. Bize, P. Laurent, M. Abgrall, H. Marion, I. Maksimovic, L. Cacciapuoti et al., "Einstein, cold atom clocks and tests of fundamental theories," J. Phys. B: At. Mol. Opt. Phys. 38, S449-S468 (2005).

${ }^{29}$ T. M. Fortier, M. S. Kirchner, F. Quinlan, J. Taylor, J. C. Bergquist, T. Rosenband, N. Lemke, A. Ludlow, Y. Jiang, C. W. Oates, and S. A. Diddams, "Generation of ultrastable microwaves via optical frequency division," Nat. Photonics 5, 425-429 (2011).

${ }^{30}$ P. L. Stanwix, M. E. Tobar, P. Wolf, M. Susli, C. R. Locke, E. N. Ivanov et al., "Test of lorentz invariance in electrodynamics using rotating cryogenic sapphire microwave oscillators," Phys. Rev. Lett. 95, 040404 (2005).

${ }^{31}$ J. G. Hartnett, C. R. Locke, E. N. Ivanov, M. E. Tobar, and P. L. Stanwix, "Cryogenic sapphire oscillator with exceptionally high long-term frequency stability,” Appl. Phys. Lett. 89, 203513 (2006).

${ }^{32}$ M. E. Tobar, E. N. Ivanov, P. L. Stanwix, J. M. Le Floch, and J. G. Hartnett, "Rotating odd-parity Lorentz invariance test in electrodynamics," Phys. Rev. D 80(12), 125024 (2009).

${ }^{33}$ J. M. Le Floch, R. Bara, J. G. Hartnett, M. E. Tobar, D. Mouneyrac, D. Passerieux, D. Cros, J. Krupka, P. Goy, and S. Caroopen, "Electromagnetic properties of polycrystalline diamond from $35 \mathrm{~K}$ to room temperature and microwave to terahertz frequencies," J. Appl. Phys. 109, 094103 (2011).

${ }^{34}$ J. M. Le Floch, F. Houndonougbo, V. Madrangeas, D. Cros, M. GuillouxViry, and W. Peng, "Thin film materials characterisation using TE modes cavity,” J. Electromagn. Waves Appl. 23, 549-559 (2009).

${ }^{35}$ B. Riddle, J. Baker-Jarvis, and M. D. Janezic, "Microwave characterization of semiconductors with a split-cylinder cavity," Meas. Sci. Technol. 19, 115701 (2008).

${ }^{36}$ R. G. Geyer, B. Riddle, J. Krupka, and L. A. Boatner, "Microwave dielectric properties of single-crystal quantum paraelectrics $\mathrm{KTaO}_{3}$ and $\mathrm{SrTiO}_{3}$ at cryogenic temperatures," J. Appl. Phys. 97, 104111 (2005).
${ }^{37}$ T. Kolodiazhnyi, G. Annino, and T. Shimada, "Intrinsic limit of dielectric loss in several $\mathrm{Ba}\left(\mathrm{B}^{\prime}{ }_{1 / 3} \mathrm{~B}^{\prime \prime}{ }_{2 / 3}\right) \mathrm{O}_{3}$ ceramics revealed by the whisperinggallery mode technique,” Appl. Phys. Lett. 87, 212908 (2005).

${ }^{38} \mathrm{P}$. Koras, L. Usydus, and J. Krupka, "Automatic split post dielectric setup for measurements of substrates and thin conducting and ferroelectric films," Ferroelectrics 434(1), 113-120 (2012).

${ }^{39}$ U. C. Hasar and O. Simsek, "A calibration-independent microwave method for position-insensitive and nonsingular dielectric measurements of solid materials," J. Phys. D: Appl. Phys. 42, 075403 (2009).

${ }^{40}$ A. A. Barannik, N. T. Cherpak, Y. V. Propenko, Y. F. Filipov, E. N. Shaforost, and I. A. Shipilova, "Two-layered disc quasi-optical dielectric resonators: Electrodynamics and application perspectives for complex permittivity measurements of lossy liquids," Meas. Sci. Technol. 18, 22312238 (2007).

${ }^{41}$ A. A. Barannik, N. T. Cherpak, and D. E. Chuyko, "Q-factor measurement of quasi-optical dielectric resonators under conditions of the whispering gallery mode degeneration removal," IEEE Trans. Instrum. Meas. 55(1), 70-73 (2006).

${ }^{42}$ T. W. Hänsch, "Passion for precision," Ann. Phys. 15(9), 627-652 (2006).

${ }^{43}$ M. D. Janezic, E. F. Kuester, and J. Baker-Jarvis, "Broadband complex permittivity measurements of dielectric substrates using split-cylinder resonator," in IEEE MTT-S International Microwave Symposium, Fort Worth, Texas, 6-11 June 2004, pp. 1817-1820.

${ }^{44}$ N. Nakayama, A. Fukuura, and M. Nishimura, "Millimetre-wave measurement of complex permittivity using dielectric rod resonator excited by NRD-guide," IEEE Trans. Microwave Theory Tech. 51(1), 170-177 (2003).

${ }^{45}$ J. Molla, "A dielectric property measurement system for this samples based on a resonant cavity with 3 dielectric regions," Meas. Sci. Technol. 13, 50-58 (2002).

${ }^{46}$ O. Piquet, D. Cros, S. Verdeyme, P. Guillon, and M. E. Tobar, "New design of high-Q sapphire resonator with distributed Bragg reflector," in IEEE MTT-S International Microwave Symposium, Seattle, Washington, 2-7 June 2002.

${ }^{47}$ J. Krupka, K. Derzakowski, M. E. Tobar, J. Hartnett, and R. G. Geyerk, "Complex permittivity of some ultralow loss dielectric crystals at cryogenic temperatures, Meas. Sci. Technol. 10, 387-392 (1999).

${ }^{48}$ E. K. Moser and K. Naishadham, "Dielectric resonators as microwave characterization tools," IEEE Trans. Appl. Supercond. 7(2), 2018-2021 (1997).

${ }^{49}$ A. N. Luiten, A. G. Mann, and D. G. Blair, High-resolution measurement of temperature-dependance of the Q, coupling and resonant frequency of a microwave resonator," Meas. Sci. Technol. 7, 949-953 (1996).

${ }^{50}$ P. Siyushev, V. Jacques, I. Aharonovich, F. Kaiser, T. Müller, L. Lombez, M. Atatüre, S. Castelletto, S. Prawer, F. Jelezko, and J. Wrachtrup, "Lowtemperature optical characterization of a near-infrared single-photon emitter source in nanodiamonds," New J. Phys. 11, 113029 (2009).

${ }^{51}$ J. Molla, A. Ibarra, J. Margineda, J. M. Zamarro, and A. Hernández, "Dielectric property measurement system at cryogenic temperatures and microwave frequencies," IEEE Trans. Instrum. Meas. 42(4), 817-821 (1993).

${ }^{52}$ J. M. Le Floch, J. D. Anstie, M. E. Tobar, J. G. Hartnett, P. Y. Bourgeois, and D. Cros, "Whispering modes in anisotropic and isotropic dielectric spherical resonators," Phys. Lett. A 359, 1-7 (2006).

${ }^{53}$ M. E. Tobar and A. G. Mann, "Resonant frequencies of higher order modes in cylindrical anisotropic dielectric resonators," IEEE Trans. Microwave Theory Tech. 39, 2077-2082 (1991).

${ }^{54} \mathrm{~J}-\mathrm{M}$. Le Floch, Ph.D. thesis, Université de Limoges and The University of Western Australia, France and Australia, 2007, see http://epublications. unilim.fr/theses/2007/le-floch-jean-michel/le-floch-jean-michel.pdf.

${ }^{55}$ O. Piquet, Ph.D. thesis, Université de Limoges, France, 2002.

${ }^{56}$ J. G. Hartnett, M. E. Tobar, J-M. Le Floch, J. Krupka, and P-Y. Bourgeois, "Anisotropic paramagnetic susceptibility of crystalline ruby at cryogenic temperature," Phys. Rev. B 75, 024415 (2007).

${ }^{57}$ P-Y. Bourgeois, Y. Kersalé, N. Bazin, M. Chaubet, and V. Giordano, "A cryogenic open-cavity sapphire reference oscillator with low spurious mode density," IEEE Trans. Ultrason., Ferroelect., Frequency Control. 51(10), 1232-1239 (2004).

${ }^{58}$ J. D. Anstie, J. G. Hartnett, M. E. Tobar, J. Winterflood, D. Cros, and J. Krupka, "Characterisation of a spherically symmetric fused-silica-loaded cavity microwave resonator," Meas. Sci. Tech. 14, 286-293 (2003).

${ }^{59}$ O. Di Monaco, Y. Kersalé, and V. Giordano, "Resonance degeneration and spurious mode suppression in a cryogenic whispering gallery mode sapphire resonator," IEEE Microwave Guided Wave Lett. 10(9), 368-370 (2000). 
${ }^{60}$ D. C. Aveline, L. Baumgartel, B. Ahn, and N. Yu, "Focused ion beam engineered whispering gallery mode resonators with open cavity structure," Opt. Express 20(16), 18091-18096 (2012).

${ }^{61}$ Y. F. Gui, W. B. Dou, P. G. Su, and K. Yin, "Improvement of open resonator technique for dielectric measurement at millimetre wavelengths," IET Microwaves Antennas Propag. 3,(7), 1036-1043 (2009).

${ }^{62} \mathrm{G}$. Annino, M. Cassettari, and M. Martinelli, "Open nonradiative cavities as millimetre wave single-mode resonators," Rev. Sci. Instrum. 76(6), 064702 (2005).

${ }^{63}$ W. W. Hansen, “A type of electrical resonator," J. Appl. Phys. 9, 654-663 (1938).

${ }^{64}$ W. W. Hansen, "On the resonant frequency of closed concentric lines," J. Appl. Phys. 10, 38-45 (1939).

${ }^{65}$ K. Fujisawa, "General treatment of klystron resonant Cavities," IRE Trans. Microwave Theory Tech. 6, 344-358 (1958).

${ }^{66} \mathrm{E}$. Rivier and M. Verge-Lapisardi, "Lumped parameters of a reentering cylindrical cavity," IEEE Trans. Microwave Theory Tech. 19, 309-314 (1971).

${ }^{67}$ M. Jaworski, "On the resonant frequency of a reentrant cylindrical cavity," IEEE Trans. Microwave Theory Tech. 26, 256-260 (1978).

${ }^{68}$ M. B. Kelly and A. J. Sangster, "Design of cylindrical reentrant cavity resonators," IEE Colloquium on Effective Microwave CAD No. 377, 1997.

${ }^{69}$ D. P. Howson, "Wide-gap re-entrant cavity resonators for $900-1500 \mathrm{MHz}$," in IEE Proceeding on Microwaves Optics and Antennas, December 1984, p. 131.

${ }^{70}$ L. J. Mittoni, N. P. Linthorne, A. G. Mann, and D. G. Blair, "Optimization of superconducting re-entrant cavities for transducer applications," J. Phys. D: Appl. Phys. 26, 804-809 (1993).

${ }^{71}$ K. Tsubono, M. Ohashi, and H. Hirakawa,"Parametric transducer for gravitational radiation detector,” Jpn. J. Appl. Phys. 25, 622-626 (1986).

${ }^{72}$ M. F. Bocko, W. W. Johnson, and V. Iafolla, "A RF superconducting electromechanical transducer for gravitational wave antennas," IEEE Trans. Magn. 25, 1358-1361 (1989).

${ }^{73}$ A. Francik, "The microwave autodyne Gunn oscillator as a simple detector of EPR signal for food-irradiation dosimeter," in Proceedings of the International Conference on Microwaves, Radar and Wireless Communications, Gdansk, Poland, 20-22 May 2002, pp. 103-106.

${ }^{74}$ C. Simon, M. Luong, S. Chel, O. Napoly, J. Novo, D. Roudier, N. Rouviere, N. Baboi, N. Mildner, and D. Nolle, "Performance of a reentrant cavity beam position monitor," Phys. Rev. Spec. Top. - Accel. Beams 11, 082802 (2008).

${ }^{75}$ A. J. C. Vieira, P. R. Herczfeld, A. Rosen, M. Ermold, E. E. Funk, W. D. Jemison, and K. J. Williams, "A mode-locked microchip laser optical transmitter for fiber radio," IEEE Trans. Microwave Theory Tech. 49, 1882-1887 (2001).

${ }^{76}$ F. Bordoni, L. Yinghua, B. Spataro, F. Feliciangeli, F. Vasarelli, G. Cardarilli, B. Antonini, and R. Scrimaglio, "A microwave scanning surface harmonic microscope using a re-entrant resonant cavity," Meas. Sci. Technol. 6, 1208-1214 (1995).

${ }^{77}$ J. Baker-Jarvis, R. G. Geyer, J. H. Grosvenor, M. D. Janezic, C. A. Jones, B. Riddle, C. M. Weil, and J. Krupka, "Dielectric characterization of lowloss materials," IEEE Trans. Dielectr. Electr. Insul. 5, 571-577 (1998).

${ }^{78}$ A. Kaczkowski and A. Milewski, "High-Accuracy wide-range measurement method for determination of complex permittivity in reentrant cavity. A: Theoretical-analysis of the method," IEEE Trans. Microwave Theory Tech. 28, 225-228 (1980).

${ }^{79}$ J-M. Le Floch, Y. Fan, M. Aubourg, D. Cros, N. C. Carvalho, Q. Shan, J. Bourhill, E. N. Ivanov, G. Humbert, V. Madrangeas, and M. E. Tobar, "Rigorous analysis of highly tunable cylindrical transverse magnetic mode re-entrant cavities,” Rev. Sci. Instrum. 84(12), 125114 (2013).

${ }^{80}$ S. K. Remillard, A. Hardaway, B. Mork, J. Gilliland, and J. Gibbs, "Using a re-entrant microwave resonator to measure and model the dielectric breakdown electric field of gases," Prog. Electromagn. Res. B 15, 175-195 (2009).

${ }^{81}$ A. A. Barannik, S. A. Bunyaev, N. T. Cherpak, Y. V. Propenko, A. A. Kherchenko, and S. A. Vitusevich, "Whispering gallery mode hemisphere dielectric resonators with impedance plane, IEEE Trans. Microwave Theory Tech. 58(10), 2682-2691 (2010).

${ }^{82}$ J. G. Hartnett, D. Mouneyrac, J-M. Le Floch, J. Krupka, M. E. Tobar, and D. Cros, "Modified permittivity observed in bulk gallium arsenide and gallium phosphide sampled at $50 \mathrm{~K}$ using whispering gallery mode method," Appl. Phys. Lett. 93(6), 062105 (2008).

${ }^{83}$ J. G. Hartnett, M. E. Tobar, E. N. Ivanov, and J. Krupka, "Room temperature measurement of the anisotropic loss tangent of sapphire using the whispering gallery mode technique," IEEE Trans. Ultrason., Ferroelect., Frequency Control. 53, 34-38 (2006).

${ }^{84}$ A. A. Barannik, Y. V. Prokopenko, Y. F. Filipov, N. T. Cherpak, and I. V. Korotash, "Q-factor of a millimeter-wave sapphire disk resonator with conductive end plates," Tech. Phys. 48(5), 621-625 (2003).

${ }^{85}$ K. Sudheendran, D. Pamu, M. Ghanashyam Krishna, and K. C. James Raju, "Determination of dielectric constant and loss of high-K thin films in the microwave frequencies, Measurement 43(4), 556-562 (2010).

${ }^{86}$ P. Queffelec, V. Laur, A. Chevalier, J-M. Le Floch, D. Passerieux, D. Cros, V. Madrangeas, A. Le Febvrier, S. Deputier, M. Guilloux-Viry, G. Houzet, T. Lacrevaz, C. Bermond, and B. Flechet, "Intercomparison of permittivity measurement techniques for ferroelectric thin layers," J. Appl. Phys. 115(2), 024103 (2014).

${ }^{87}$ M. E. Tobar, E. N. Ivanov, J. G. Hartnett, and D. Cros, "High-Q frequency stable dual-mode whispering gallery sapphire resonator," in IEEE MTT$S$ International Microwave Symposium, Phoenix, Arizona, 20-25 May 2001, pp. 205-208.

${ }^{88}$ B. W. Hakki and P. D. Coleman, "A dielectric resonator method of measuring inductive capacities in the millimetre range," IEEE Trans. Microwave Theory Tech. 8, 402-410 (1960).

${ }^{89}$ W. E. Courtney, "Analysis and evaluation of a method pf measuring the complex permittivity and permeability of microwave insulators," IEEE Trans. Microwave Theory Tech. 18, 476-485 (1970).

${ }^{90}$ IEC 61338, Sections 1-1, 1-2, and 1-3 (1996-2003), Waveguide type dielectric resonators: Part 1 . General information and test conditionsSection 3. Measurement method of complex permittivity for dielectric resonator materials at microwave frequency, see www.iec.ch.

${ }^{91}$ J. Krupka, "Frequency domain complex permittivity measurements at microwave frequencies," Meas. Sci. Technol. 16, R1-R16 (2005).

${ }^{92}$ Y. Kobayashi and M. Katoh, "Microwave measurement of dielectric properties of low-loss materials by the dielectric rod resonator method," IEEE Trans. Microwave Theory Tech. 33, 586-592 (1985).

${ }^{93}$ J. Breeze, J. Krupka, A. Centeno, and N. McN. Alford, "Temperaturestable and high-Q factor TiO2 Bragg reflector resonator,” Appl. Phys. Lett. 94(8), 082906 (2009).

${ }^{94}$ Kagomiya, Y. Matsuda, K. Kakimoto, and H. Ohsato, "Microwave dielectric properties of YAG," Ferroelectrics 387(1), 1-6 (2009).

${ }^{95}$ Y. Zhou, Z. Yue, and L. Li, "Preparation and microwave dielectric properties of $\mathrm{TiO}_{2}$-doped YAG ceramics," Ferroelectrics 407(1), 69-74 (2010).

${ }^{96}$ N. A. Shtin, J. M. Lopez Romero, and E. Prokhorov, "Theory of fundamental microwave absorption in sapphire ( $\alpha$-Al203)," J. Appl. Phys. 106, 104115 (2009).

${ }^{97}$ X. Aupi, J. Breeze, N. Ljepojevic, L. J. Dunne, N. Malde, A.-K. Axelsson, and N. McN. Alford, "Microwave dielectric loss in oxides: Theory and experiment," J. Appl. Phys. 95(5), 2639-2645 (2004).

${ }^{98}$ M. Kusunoki, M. Inadomaru, S. Ohshima, K. Aizawa, M. Mukaida, M. Lorenz, and H. Hochmuth, "Dielectric loss tangent of sapphire single crystal produced by edge-defined film-fed growth method," Physica C 377, 313-318 (2002).

${ }^{99}$ N. McN. Alford, J. Breeze, X. Wang, S. J. Penn, S. Dalla, S. J. Webb, N. Ljepojevic, and X. Aupi, "Dielectric loss of oxide single crystals and polycrystalline analogues from 10 to 320K," J. Eur. Ceram. Soc. 21, 26052611 (2001).

${ }^{100}$ S. N. Buckley, P. Agnew, and G. P. Pells, "Cryogenic dielectric properties of sapphire at 2.45GHz, J. Phys. D: Appl. Phys. 27, 2203-2209 (1994).

${ }^{101}$ S. Castelletto, J. P. Harrison, L. Marseglia, A. C. Stanley-Clarke, B. C. Gibson, B. A. Fairchild, J. P. Hadden, Y-L. D. Ho, M. P. Hiscocks, K. Ganesan, S. T. Huntington, F. Ladouceur, A. D. Greentree, S. Prawer, J. L. O'Brien, and J. G. Rarity, "Diamond-based structures to collect and guide light," New J. Phys. 13, 025020 (2011).

${ }^{102}$ P. St and J. Russell, "Photonic-crystal fibers," J. Ligthwave Tech. 24, 4729-4749 (2006)

${ }^{103}$ G. Humbert, J-M. Le Floch, D. Mouneyrac, D. Ferachou, M. Aubourg, M. Tobar et al., "Hollow-core resonator based on out-of-plane twodimensional photonic band-gap crystal cladding at microwave frequencies," Appl. Phys. Lett. 96, 051108 (2010).

${ }^{104}$ D. Ferachou, G. Humbert, J-M. Le Floch, M. Aubourg, J-L. Auguste, M. E. Tobar, D. Cros, and J-M. Blondy, "Compact hollow-core photonic band gap resonator with optimized metallic cavity at microwave frequencies," Electon. Lett. 47, 805-U92 (2011).

${ }^{105}$ F. Couny, H. Sabert, P. J. Roberts, D. P. Williams, A. Tomlinson, B. J. Mangan, J. C. Knight, T. A. Birks, and P. St. J. Russel, "Visualizing the photonic band gap in hollow core photonic crystal fibers," Opt. Express 13(2), 558-563 (2005). 